\title{
Close Pairs of Field Galaxies in the CNOC1 Redshift Survey
}

\author{
D. R. Patton ${ }^{1}$, C. J. Pritchet ${ }^{1,2}$, H. K. C. Yee ${ }^{2,3}$, E. Ellingson ${ }^{2,4}$, and R. G. Carlberg ${ }^{2,3}$
}

Received 1996 June 3; accepted _

\footnotetext{
${ }^{1}$ Department of Physics and Astronomy, University of Victoria, PO Box 3055, Victoria, BC, V8W 3P6, Canada.

${ }^{2}$ Visiting Astronomer, Canada-France-Hawaii Telescope, which is operated by the National Research Council of Canada, le Centre National de Recherche Scientifique, and the University of Hawaii.

${ }^{3}$ Department of Astronomy, University of Toronto, 60 St. George Street, Toronto, ON, M5S 3H8, Canada.

${ }^{4}$ Center for Astrophysics and Space Astronomy, University of Colorado, Campus Box 389, Boulder, CO 80309-0389.
} 


\begin{abstract}
A redshift sample of 545 field galaxies is used to examine how the galaxy merger rate changes with redshift, and how mergers affect the observed properties of galaxies in close pairs. Close pairs are defined as those with projected separations less than $20 h^{-1} \mathrm{kpc}$. At a mean redshift of 0.33 , it is found that $7.1 \pm 1.4 \%$ of galaxies are in close physical pairs, compared to $4.3 \pm 0.4 \%$ locally. The merger rate is estimated to change with redshift as $(1+z)^{2.8 \pm 0.9}$. These results are shown to be consistent with previous close pair studies, and provide one of the strongest constraints to date on the redshift dependence of the merger rate.

As with earlier studies, no significant differences between the mean properties of paired and isolated galaxies are detected. However, using the subset of confirmed close physical pairs, those which appear to be undergoing interactions or mergers (based on their images) are found to have galaxies with strong emission lines and very blue rest-frame colors. In addition, these pairs have low relative velocities, increasing the likelihood that the galaxies will merge. We interpret this as the first clear evidence of merger-induced star formation occurring in field galaxies at this redshift.
\end{abstract}

Subject headings: galaxies : evolution — galaxies : interactions - galaxies : starburst - surveys 


\section{INTRODUCTION}

Accumulating evidence indicates that galaxy populations have undergone significant evolution in the recent past. This initially became apparent with the excess number counts of faint blue galaxies (Tyson 1988). However, various redshift surveys (Broadhurst, Ellis, \& Shanks 1988; Colless et al. 1990, 1993) showed the redshift distribution of faint galaxies to be indistinguishable from no-evolution model predictions. The picture that is emerging from recent observations is one in which the number density of $L^{*}$ galaxies was higher at moderate redshift (Lilly et al. 1995, Lin et al. 1996). This can be explained by the presence of star-bursting dwarf galaxies, which have since faded. At present, it is unclear what is driving this process. One possible explanation is galaxy-galaxy merging (Rocca-Volmerange

\& Guiderdoni 1990; Broadhurst, Ellis, \& Glazebrook 1992; Carlberg \& Charlot 1992). Merging is expected to make galaxies brighter and somewhat bluer, due to enhanced star formation (Larson \& Tinsley 1978), and implies that galaxies were less massive in the past. However, the importance of merging in the observed evolution of galaxies remains an open question.

Galaxy merging affects a small fraction of galaxies at the present epoch. If the rate of merging remains constant, galaxy masses at $z=1$ will be reduced to about two thirds of their values at the present epoch for $\Omega=1$ (Carlberg 1995). However, there are a number of reasons to believe that the merger rate was significantly higher in the past. First, since the universe is expanding, galaxies were closer together in the past, so naturally merging would have been more prevalent. Also, recent Hubble Space Telescope (HST) imaging of galaxies at moderate redshift (e.g. Griffiths et al. 1994 ; Driver, Windhorst, \& Griffiths 1995; Glazebrook et al. 1996) reveals a relatively high proportion of galaxies which are morphologically anomalous, often exhibiting signs of interactions. Finally, a number of studies of close pairs of galaxies (e.g. Zepf \& Koo 1989; Carlberg, Pritchet, \& Infante 
1994 [hereafter CPI]; Yee \& Ellingson 1995 [hereafter YE]) have attempted to measure the

change in the merger rate with redshift; such studies consistently find a significant increase with redshift.

The CNOC (Canadian Network of Observational Cosmology) cluster redshift survey (hereafter CNOC1, see Yee, Ellingson, \& Carlberg 1996 [hereafter, YEC]; and Carlberg et al. 1996) was undertaken primarily to study galaxy clusters. However, a wealth of information on field galaxies was also compiled. This study utilizes the CNOC1 catalogs to investigate the properties of close pairs of field galaxies, and to place tighter constraints on the evolution of the merger rate. The availability of large numbers of redshifts represents an important improvement over previous studies of close pairs.

In $\S 2$, the CNOC1 observations will be briefly summarized. Section 3 describes the selection procedure used to identify an unbiased sample of field galaxies. The pair fractions are determined in $\S$ 田 and $\S$, and the properties of galaxies which are paired and isolated are compared in $\S$ 6. The redshift dependence of the galaxy merger rate is calculated in $\S$ 1. The sample is divided up into redshift bins in $\S 8$ to investigate possible trends with redshift. We focus on the subset of confirmed physical pairs in $\S$ 9. Finally, the results and ensuing implications are discussed in $\S 10$. When necessary, we assume $H_{0}=100 \mathrm{~km} \mathrm{~s}^{-1} \mathrm{Mpc}^{-1}(h=1)$ and $q_{0}=0.5$ throughout.

\section{OBSERVATIONS}

Observations for the CNOC1 redshift survey were carried out using a multi-object spectrograph (MOS/SIS) at the Cassegrain focus of CFHT. The data catalogs consist of photometry (in Gunn $g$ and $r$ ) in the regions of 16 rich clusters of galaxies, lying at redshifts of $0.18-0.55$. Survey fields of up to $6 \mathrm{Mpc}$ per cluster were covered, with the outer regions 
being dominated by the field population. Redshifts have been obtained for roughly half of the brighter galaxies, of which $\sim 50 \%$ are confirmed field galaxies; in the outer regions of the clusters, this fraction rises to $\sim 80 \%$. For a complete description of the CNOC1 observational strategy and data reduction techniques, see YEC.

\subsection{Photometric Data}

The basic sample consists of a catalog of photometry in Gunn $g$ and $r$, which is complete to a magnitude of $r=23.0$ (and fainter for most frames). For the purposes of this study, a uniform photometric limiting magnitude of $r=23.0$ is applied. The entire

photometric survey is $100 \%$ complete to this limit, and most frames are complete to $r \sim$ 23.5. Star-galaxy classification has been performed, and is considered to be very reliable as faint as $r \sim 22.5$. Misclassification (mostly of galaxies as stars) is $\lesssim 10 \%$ at $r \sim 23.5$ for most images, which is fainter than the limiting magnitude used throughout this analysis. This gives us a catalog with 14831 galaxies covering $\sim 0.66 \mathrm{deg}^{2}$.

\subsection{Spectroscopic Data}

The CNOC1 catalog contains redshifts for $\sim 2600$ faint galaxies, of which roughly half are field galaxies. Redshifts and rough spectral classification were performed using the cross-correlation techniques described by Ellingson \& Yee (1994). The spectroscopic completeness (defined as the fraction of objects with redshifts) varies from region to region, depending on the projected number density of cluster members and the number of MOS masks taken for a given field (ranging from 1-3). The cumulative spectroscopic completeness ranges from $\sim 50 \%$ at $r=21.5$ to $\sim 17 \%$ at $r=23.0$ (here cumulative refers to all galaxies brighter than the given apparent magnitude). Each redshift in the 
catalog has been assigned a "significance parameter" $\mathrm{R}$, which is a measure of the strength of the redshift cross-correlation. We must consider two types of uncertainty in redshift measurements; identification error and velocity error. The former arises when the wrong peak in the cross-correlation is selected, and can result in catastrophic redshift errors. Three quarters of the redshifts have either emission-line spectra with $\mathrm{R}>5$ or absorption-line spectra with $\mathrm{R}>4$; such redshifts are considered to be very secure ( $\gtrsim 99 \%$ confidence, see YEC). The confidence level for the remaining objects drops to $\sim 95 \%$. As an extra precaution against identification errors, all redshifts with $\mathrm{R}<3$ ( $\sim 2 \%$ of the sample) are excluded from the analysis. This leaves us with redshifts for 2530 galaxies. Once a redshift has been identified, the uncertainty in velocity can be estimated in several ways. The most rigorous method is to use redundant observations; this yields a typical estimated uncertainty of $\sim 130 \mathrm{~km} / \mathrm{s}$. Redshift errors are discussed further in YEC.

\section{SAMPLE IDENTIFICATION}

\subsection{The Primary Redshift Sample}

In order to study close pairs, we desire a sample of field galaxies (with redshifts) which is unbiased with respect to galaxy type and does not favour the identification of paired galaxies over isolated galaxies. The following sections describe the restrictions imposed to identify such a sample.

\subsubsection{Excluding Cluster Members}

Since we are interested in the close pair properties of field galaxies, we must first remove all cluster galaxies from the primary redshift sample. Eliminating known cluster members is a fairly straightforward procedure. For each cluster, a weighted mean redshift 
has been identified, along with corresponding upper and lower redshift limits. These limits

were derived by Carlberg et al. (1996), using a manually iterated procedure which depends on each cluster's measured velocity dispersion. A similar (but slightly less strict) constraint is to uniformly exclude all galaxies with velocities within $3000 \mathrm{~km} / \mathrm{s}$ of each cluster's median velocity (the main results of this study are unchanged by imposing this criterion instead). After this restriction, we are left with a sample of 1257 field galaxies.

\subsubsection{Excluding Field Galaxies with Potential Redshift Biases}

In the original survey, band-limiting filters were used to reduce overlap of spectra, and hence to maximize the number of cluster redshifts obtained (see YEC). As a result, the limited spectral range associated with each filter imposes a restriction on the range of redshifts for which field galaxies can be consistently identified. Specifically, the lower limit is set by the ability to detect the $[\mathrm{O}$ II $] \lambda 3727$ line within $50 \AA$ of the blue edge of the spectra, while the upper limit reflects the redshift at which the $4000 \AA$ break is within 150 $\AA$ of the red edge. The appropriate field redshift ranges can be found in Table 2 of YEC. All field galaxy redshifts within the appropriate redshift range are expected to be unbiased with respect to the redshift acquisition procedure, and hence are included in the primary sample. This leaves 797 field galaxies.

\subsubsection{Excluding the Faintest Field Galaxies}

The CNOC1 mask design algorithm selects proportionately fewer faint galaxies, in order to maximize the number of cluster galaxies obtained. In addition, fainter galaxies have spectra with lower signal-to-noise ratios, making it more difficult to extract reliable redshifts. To compensate for this, a magnitude selection function was measured, which 
estimates the spectroscopic completeness (the fraction of galaxies with redshifts) as a function of magnitude (see YEC). All galaxies with measured redshifts were assigned a weight, which is the inverse of the selection function for that magnitude, and for that particular region. To ensure that the primary field galaxy sample is unbiased, we wish to identify the range in apparent magnitude within which the spectroscopic completeness is at least $25 \%$. Using the weights of all galaxies with redshifts, we determined this magnitude range for each field. As illustrated in Figure 1, this amounts to identifying a faint limiting magnitude only, since the completeness is greater than $25 \%$ for the brightest galaxies. All field galaxies which are fainter than this spectroscopic limiting magnitude are excluded from the primary sample.

\subsubsection{Avoiding the Clusters}

After all such restrictions have been made, we are left with an unbiased primary sample consisting of 572 field galaxies. However, there remains the danger of a given field galaxy being superimposed on the central region of a dense cluster of galaxies. In this case, the probability of a close companion being an optical superposition is greatly increased. To avoid this situation, we wish to exclude from the primary sample all field galaxies lying in regions which contain large numbers of cluster galaxies. This exclusion was carried out by computing the number density of galaxies around each field galaxy, within a somewhat arbitrarily-defined radius of $1^{\prime}$. This radius was chosen to be large enough to minimize the

influence of close $\left(<20 \mathrm{~h}^{-1} \mathrm{kpc}\right)$ companions, while remaining small enough to detect local number density effects, such as the presence of the core of a cluster. It is desirable to use a faint limiting magnitude (e.g. $r=23.0$ ) to provide good statistics. However, to maximize sensitivity to the presence of cluster members, it is better to use a limiting magnitude which is closer to $M^{*}$. For the CNOC1 clusters, $M^{*}$ typically corresponds to $r \sim 20$, and the 
faintest is $r \sim 21.7$ (here, we have assumed $M_{r}^{*}=-20.6$ mag for early-type galaxies from local galaxy luminosity functions (LFs) [eg., King \& Ellis 1985]). At fainter magnitudes, the slope of the cluster galaxy LF flattens out relative to that of field galaxies, decreasing the fraction of cluster members. For this calculation only, a limiting magnitude of $r=$ 22.0 was selected. This includes an acceptable number of galaxies for computing the local number density, while being as close as possible to $M^{*}$.

A histogram of the number density in the region of all field galaxies is shown in Figure 2. This plot shows the existence of a high-density tail, which is expected to be those field galaxies lying in the central regions of clusters. A density threshold of 7 galaxies per square arcminute was selected, in order to eliminate most of the high-density tail while

keeping the majority of galaxies having more typical local densities. In addition, all galaxies lying within $3^{\prime}$ of a cluster center were excluded from the primary sample. All plots were checked by eye to ensure that: a) no primary galaxies remain in the central regions of clusters and b) no field galaxies lying far from the central regions are excluded from the primary sample. Using these criteria, 27 galaxies were excluded, leaving 545 galaxies in the primary sample.

\subsection{The Secondary Sample}

The initial task in a study of close pairs is to determine which galaxies have close companions. As with previous studies, we wish to look for companions which are comparable in luminosity to the primary sample, and to determine (where possible) which of these companions are physically associated. Therefore, we wish to identify a secondary sample, consisting of all galaxies (with or without redshifts) which are potential companions. In order to restrict the secondary sample to galaxies of similar luminosities, we need to impose the same restrictions in apparent magnitude as were earlier applied to the primary sample. 
Hence, we apply the spectroscopic limiting magnitudes derived in $\S 3.1 .3$ to all galaxies in the survey (with or without redshifts). The secondary sample is not distinct from the primary sample; rather, the primary sample is a subset of the secondary sample. There are 3739 galaxies in the secondary sample, of which roughly half (1972 galaxies) have redshifts.

\section{THE OBSERVED PAIR FRACTION}

In order to discuss the fraction of galaxies in close pairs, one must define what is meant by "close pair". For the purposes of this study, a close pair is defined as two galaxies with a projected separation of no more than $20 h^{-1} \mathrm{kpc}$. At this physical separation, dark galaxy halos and even visible disks will interact significantly (Carlberg 1995). N-body simulations indicate that these pairs will merge within $\sim 0.5$ Gyr (e.g. Barnes 1988), as long as the galaxies have reasonably low relative velocities. For a galaxy with a known redshift, a projected separation $\mathrm{D}$ translates into an angular separation $\theta$ using the equation

$$
\theta=\frac{D H_{0}(1+z)^{2}}{2 c(1+z-\sqrt{1+z})}
$$

(we assumed $\Omega_{0}=1$ for these calculations; at $z=0.33, \theta$ would be $\sim 6 \%$ smaller for $\Omega_{0}$ $=0.2$ ). For redshifts of $0.18-0.67$ in the primary sample, $\mathrm{D}=20 h^{-1} \mathrm{kpc}$ corresponds to separations (denoted by $\theta_{20}$ ) of 10 .'2 -5 ". 1 , with a mean separation criterion of $\sim 7^{\prime \prime}$.

To calculate the observed fraction of galaxies in close pairs, one simply counts the number of primary field galaxies which have companions within $20 h^{-1} \mathrm{kpc}$. Companions are selected from the secondary sample, and may or may not have redshifts. For the sample of 545 primary galaxies, 73 were found to have at least one close companion. A slight correction was made to take into account the fact that a small fraction of the search area is not covered by the survey (due to saturated stars, column bleeding, etc.). Using the number of companions observed (81), as compared to the number we would expect to have 
found if coverage had been complete (81.6), the expected number of galaxies with at least one companion is then 73.6, yielding a close pair fraction of $13.5 \pm 1.6 \%$ (errors assume Poisson statistics).

\subsection{Resolution Effects and the Angular Correlation Function}

Seeing for the $r$ images was typically $\sim 1^{\prime \prime}(\mathrm{FWHM})$. Hence, we are unable to resolve close pairs at or below this angular separation. To investigate empirically what effect this may have on the calculated pair fraction, we have plotted the number density of pairs versus angular separation for the entire $r \leq 23.0$ sample in Figure 3 a. Three features that stand out are: (a) at large $\left(z 7^{\prime \prime}\right)$ separations, the number density of pairs is roughly constant, as would be expected for a random distribution of galaxies; (b) there is a significant excess (over random) of close pairs at separations of a few arcseconds; and (c) there is a clear deficit of close pairs at separations $\lesssim 1^{\prime \prime} .5$.

While the excess over random is significant, an excess is expected from studies of the angular correlation function. This function, $\omega(\theta)$, measures the excess number of galaxies (over random) found at a given angular separation $\theta$, and can be accurately represented by a power law of the form $\omega(\theta)=A_{\omega} \theta^{-\delta}$ (Peebles 1980). To estimate the expected $\omega(\theta)$ for our sample, we transform our magnitude limit to the $F$ band, using the color transformation of Windhorst et al. (1991) and the average color $(g-r=0.82)$ for our sample. Assuming the canonical value of $\delta=0.8$ (Peebles 1980), the amplitude for our sample is estimated to be $A_{\omega}\left(1^{\prime}\right)=0.056$, using the observed $F$ band fits of Infante \& Pritchet (1995). For comparison, we also use their results for $\delta=0.6$ (the shallowest slope found), which gives $A_{\omega}\left(1^{\prime}\right)=0.062$. We can then compute the expected pair number density, since $\sigma_{\text {exp }}(\theta)=[1+\omega(\theta)] \sigma_{a v}$. Here, $\sigma_{\text {exp }}(\theta)$ is the expected pair number density at separation $\theta$, while $\sigma_{a v}$ is the average number density of galaxies. Note that $\sigma_{a v}$ was computed using 
the observed counts at separations of $1^{\prime}$, and dividing by $1+\omega\left(1^{\prime}\right)$. That is, the observed and predicted pair densities are normalized to agree at $\theta=1^{\prime}$. The predicted relations are plotted in Figure 3a, and we can see that the observed excess is roughly consistent with what is expected from the angular correlation function. The expected $\omega(\theta)$ is derived from a pure field sample; what effect do the superimposed CNOC1 cluster galaxies have on this? To address this issue, the analysis was repeated after excluding all known cluster members from the sample. As seen in Figure $3 \mathrm{~b}$, the agreement is similar, and indicates that the cluster galaxies do not strongly influence the observed relation.

We conclude that the observed number density of pairs is consistent with the extrapolation of the angular correlation function to small scales. We note that, while Woods, Fahlman, \& Richer (1995) find no excess over random in their photometric survey of faint pairs, their result appears to be consistent with the excess found in this study. Our Figure 3 shows that our results are clearly not consistent with no excess over random. The deficit of close pairs at very small scales is confirmed, however, and is clearly due to resolution effects. To compensate for this effect, a minimum search radius of $1^{\prime \prime}$ was implemented when computing the observed pair fraction. None of the primary field galaxies have observed companions within this radius; hence, the observed pair fraction remains the same as above. We are unable to correct for unresolved companions; however, by extrapolating $\omega(\theta)$ to the smallest scales, we would expect companions closer than $\sim 1^{\prime \prime}$ to contribute $\lesssim 5 \%$ to the observed pair fraction. This estimate is consistent with the HST pairs study by Burkey et al. (1994; hereafter BKWF), who found that only 1 out of their 25 close pairs had a projected separation of $<1^{\prime \prime}$. This effect is sufficiently small (compared to the uncertainty in the measured pair fraction) that we have chosen to ignore it. 


\subsection{Sampling Effects}

When using an incomplete redshift survey to calculate the close pair fraction, one must sample paired galaxies fairly, or else correct for unfair sampling. Although the CNOC1 survey is designed to compensate for pair-distribution selection effects (see YEC), we will demonstrate a method of determining if pairs are in fact sampled fairly. In addition, this

method will enable us to estimate the observed pair fraction in a sample which is not fairly sampled.

We begin with an idealized sample in which all redshifts are known. Then, by modelling the redshift selection procedure, one can compute what the observed pair properties of the sample would be. Suppose we start with a sample of $N$ galaxies, of which a fraction $x$ lie in apparent close pairs. We model the redshift selection as a two-step procedure. First, let $S$ be the probability of obtaining a redshift for an isolated galaxy or the first galaxy in a close pair. Then, let $R$ be the probability of obtaining a redshift for the second galaxy in a pair, assuming a redshift has already be obtained for the first. If pair selection is fair, $R=S$. Now, let us also assume that, for all galaxies for which redshifts are obtained, a fraction $P$ will be members of the primary sample (i.e. lying in the redshift range of interest). Knowing these properties, we can determine what the actual observed sample will look like. The observable properties we will use are as follows: $n p_{1}$ is the number of primary galaxies which have companions with redshifts, $n p_{0}$ is the number of primary galaxies which have companions without redshifts, $n_{0}$ is the number of primary galaxies which are isolated, and $n n z$ is the number of galaxies without redshifts. By applying the selection procedure, it follows that

$$
\begin{gathered}
n p_{1}=N x S R P, \\
n p_{0}=\frac{1}{2} N x S(2-R-S) P, \\
n_{0}=N(1-x) S P
\end{gathered}
$$




$$
n n z=\frac{1}{2} N x S(S-R)+N(1-S)
$$

We now have four independent equations, with four input parameters $(S, R, P, x)$ and four resultant observed quantities $\left(n p_{1}, n p_{0}, n_{0}, n n z\right)$. For a real sample, we can retrieve the input parameters by solving this system of equations in terms of the observables. The solution is as follows:

$$
\begin{gathered}
A=N\left(n p_{1}+n p_{0}+n_{0}\right) \\
B=n_{0}(n n z-3 N)-2 N\left(n p_{1}+n p_{0}\right), \\
C=(N-n n z)\left(n p_{1}+2 n p_{0}+2 n_{0}\right), \\
S=\frac{-B-\sqrt{B^{2}-4 A C}}{2 A} \\
R=\frac{n p_{1}(2-S)}{n p_{1}+2 n p_{0}} \\
P=\frac{n_{0}}{N(1-x) S} \\
x=\frac{n p_{1}+2 n p_{0}}{(2-S) n_{0}+n p_{1}+2 n p_{0}} .
\end{gathered}
$$

We now apply this technique to our sample, in order to determine the input parameters. However, for a real sample, we have the complicating effect that galaxies may be found in multiple systems. That is, a primary galaxy may have more than one companion. For our sample, there are 65 primary galaxies with one companion, and 8 with two companions. We take this effect into account by using the fraction of companions with redshifts to calculate $n p_{1}$ and $n p_{0}$. There are 49 companions with redshifts and 32 without; hence, $n p_{1}=44.2$ and $n p_{0}=28.8$, for a total of 73 paired primary galaxies. The number of isolated primary galaxies $\left(n_{0}\right)$ is 472 , giving a total of 545 primary galaxies. For a pure field sample, $n n z$ and $N$ are easy to determine. With the CNOC1 survey, we must keep in mind that primary galaxies are selected so as to avoid cluster centers - hence, only a subset of the area is truly surveyed for primary galaxies. Instead, we compute an effective $N$ (and effective $n n z$ ), by 
determining the ratio of primary galaxies to total number of galaxies (and total number without redshifts) in the neighbourhood of each of the primary galaxies. An annulus with inner radius of $1^{\prime}$ and outer radius of 1'.5 was used, which is representative of the sample as a whole. The total number of galaxies within these annuli is 3108, of which 1329 have no redshifts and 781 are members of the primary sample. This leads to $N=2169$ and $n n z=$ 927.

Using equations 6 - 9, we find : $S=57.1 \%, R=62.1 \%, P=43.9 \%$, and $x=13.1$

$\%$. What does this mean? First of all, $S$ and $R$ agree closely. If we define the pair sampling rate to be $R / S$, we find a value of $109 \%$. This implies that galaxies in pairs are sampled in the same way as other galaxies, demonstrating that the mask design algorithm of YEC successfully compensates for pair selection bias. Also, $x$ is in fact the observed pair fraction for all galaxies in the sample. Strictly speaking, this is not the same as the observed pair fraction for primary galaxies, but it should be fairly similar, since non-primary galaxies cover a similar (but larger) range in redshift. After correcting for incomplete area coverage (see $\S$ 母), we find an observed pair fraction of $13.2 \%$, in excellent agreement with the result found earlier in $\S$.

\section{THE PHYSICAL PAIR FRACTION}

\subsection{Companions With Redshifts}

A total of 81 companions were found, of which 49 have redshifts. However, a significant fraction of these companions could be optical superpositions. We can use the available redshifts to weed out some apparent companions which lie at significantly different redshifts from the primary galaxies. The aim here is to exclude only those galaxies which cannot be physically associated with the primary. Fortunately, choosing an appropriate cutoff 
turns out to be quite simple. For all possible pairs with 2 redshifts, 2 quantities were

calculated: projected separation $\mathrm{D}$ (in $h^{-1} \mathrm{kpc}$ ), and rest-frame velocity difference $\Delta V$ (in $\mathrm{km} / \mathrm{s}$ ). Projected separation is calculated with equation 1, using the angular separation and average redshift for the pair. These quantities are plotted in Figure 1 a, for large ranges of $\mathrm{D}$ and $\Delta V$. Points lying in the lower portion of the plot represent pairs of galaxies which are apparently close together on the sky. Of these, only those with relatively low velocity differences may in fact be physically close. From the plot, there is an obvious population of close $\left(\mathrm{D}<20 h^{-1} \mathrm{kpc}\right.$ ) physical pairs, all of which have $\Delta V<600 \mathrm{~km} / \mathrm{s}$; these are shown more clearly in Figure $4 \mathrm{~b}$. The remaining pairs have $\Delta V>4000 \mathrm{~km} / \mathrm{s}$ (note that velocity errors are at most $150 \mathrm{~km} / \mathrm{s}$, and are typically $\sim 130 \mathrm{~km} / \mathrm{s}$ ). Using a pair velocity criterion of $1000 \mathrm{~km} / \mathrm{s}$, we find that 22 companions (with redshifts) are not physically associated with the corresponding primary galaxy. Hence, $55 \%$ of the companions with redshifts are deemed to be physically associated. If we had redshifts for all galaxies in the sample, this would allow us to eliminate all optical companions.

\subsection{Companions Without Redshifts}

Even without a redshift, one can glean some information about a galaxy's distance from its color. Specifically, at any given redshift, there is a limit to how red normal galaxies are expected to be, the reddest being those of E/S0 morphological types. Any galaxies which are significantly redder than this can safely be assumed to lie at a higher redshift. Recall that the E/S0 envelope gets progressively redder with increasing redshift (cf. Koo \& Kron 1992). This is also strikingly apparent in the color-redshift distribution of the field galaxies in this study (see Figure 5). For a given field galaxy at redshift $z$, we assume that all companions lying more than 0.15 magnitudes redward of the $E / S 0$ sequence must lie at higher redshift. Note that once again we wish to eliminate only those galaxies which cannot 
be physically related, so it is best to make a conservative cut only. It turns out that none of the companions are eliminated using this restriction. However, in a larger and deeper redshift sample, this technique could be used to eliminate a significant number of optical companions.

We are left with 32 companions without redshifts. Without additional information about these galaxies, we cannot determine which companions are physically associated on a galaxy-by-galaxy basis. However, we can correct for this effect statistically, by comparing the number density of the remaining companions with the background density of galaxies without redshifts. That is, we can calculate the number of companions expected (on average) in a random distribution, and compare it to the number actually found. To estimate the number of random companions expected, the average number density of galaxies (without redshifts) near each galaxy was computed. Once again, we use the color cutoff. This was done using an annulus with an outer radius of 1'.5 arcmin and an inner radius of twice $\theta_{20}$ (typically $\sim 15^{\prime \prime}$ ). The outer radius was chosen to be large enough to properly sample the local region around the galaxy, but small enough to minimize the effects of cluster density gradients. The inner radius was chosen to be large enough to avoid the influence of the galaxy and its close companions on the number density. The number of random companions expected for a given galaxy was computed by multiplying the number density by the effective search area. Due to the resolution effects described earlier, we use annuli with inner radii of $1^{\prime \prime}$ and outer radii of $\theta_{20}$. Any excess over random was then attributed to the presence of physically associated companions.

The total number of random companions (without redshifts) expected is $16.8 \pm 0.4$, as compared to 32 found. This optical fraction (53\%) is consistent with that found for companions with redshifts $(45 \%)$, demonstrating that the statistical correction for optical companions is reliable (in fact, we expect the optical fraction to be slightly higher for 
companions without redshifts, since galaxies outside the primary redshift limits are less likely to have redshifts). It follows that the overall fraction of companions (with or without redshifts) which are physical is $52.2 \pm 8.6 \%$. Therefore, the fraction of galaxies which are in close physical pairs is $7.1 \pm 1.4 \%$.

\section{PROPERTIES OF GALAXIES IN CLOSE PAIRS}

In order to investigate the properties of galaxies in close pairs, we wish to compare the "paired" galaxies (those in the primary sample with companions of comparable luminosity) with galaxies which are not in pairs. For this part of the analysis, a "paired" galaxy is defined as one which has at least one companion which may be physically associated. Hence, known optical companions will be discarded. In identifying an isolated sample, we note that some galaxies have companions which are too faint to be included in the secondary sample. If we include these galaxies in the sample of galaxies not in pairs, we may be smearing out any observed property differences. Instead, we define the "isolated" sample to be those galaxies in the primary sample which have no companions brighter than the limiting magnitude of the entire sample $(r=23.0)$. Galaxies with faint companions only (hereafter designated "paired [faint]") will be included in the ensuing analysis for completeness, but we will focus on comparing the paired and isolated samples. The mean properties are summarized in Table 1 (unless otherwise stated, errors quoted are errors in the mean). The results of various Kolmogorov-Smirnov (K-S) tests are presented in Table 2. The K-S test statistic given indicates the significance level for the hypothesis that two sets of data are drawn from the same distribution. A small significance level indicates that two distributions have significantly different cumulative distribution functions. 


\subsection{Redshifts}

Of primary interest is the redshift distribution of the two samples. YE found that the average redshifts of their paired and isolated samples were identical to within $\frac{\Delta z}{z} \lesssim 0.1$, implying that both populations have similar characteristic luminosities. With about 5 times as many redshifts, we can place even tighter constraints on this relation. The average redshift for our paired sample is statistically equivalent to that of the isolated sample. We

conclude that the average redshifts of the two samples are identical to within $\frac{\Delta z}{z} \lesssim 0.05$. A K-S test and the visual appearance of the redshift histograms (see Figure 6) are consistent with this conclusion.

\subsection{Galaxy Classification}

Each field galaxy was classified using its $g-r$ color and redshift. The basic method was to use a fit to the color $k$-corrections of YEC, who convolved typical galaxy spectra from Coleman, Wu, \& Weedman (1980; hereafter CWW) with the filters used in the observations. The color-redshift relations corresponding to elliptical, Sbc, Scd, and Im galaxies are plotted in Figure 1. Each primary galaxy's location on this plot was used to estimate the most appropriate type classification by using the sequence with the closest color at the galaxy's redshift. The relative proportions of early and late-type galaxies were determined for both the paired and isolated samples. The fraction of late-type $(\mathrm{Scd}+\mathrm{Im})$ galaxies is slightly lower for the paired sample, but the difference is not statistically significant. The corresponding K-S test result is consistent with this conclusion.

We can perform an independent check on this result, using the spectral classification given in the CNOC1 catalogs. Each galaxy has been assigned a spectral type based on the template giving the highest redshift cross-correlation value. Galaxies are identified as either 
early-type, Sbc, or emission-line galaxies. This determination is independent of color, and hence provides a good comparison with the classifications determined above. We find that the fraction of emission-line galaxies is slightly lower for the paired sample, but again the difference is not significant, as demonstrated by the K-S test. Hence, neither spectral nor color classification reveals any measurable differences between paired and isolated galaxies.

\subsection{Colors}

Since we might expect galaxies in close pairs to have different colors than other galaxies, we compare the observed color distributions of the two sets of data. The mean $g-r$ colors were found to agree quite closely, and a K-S test confirms this. This result has also been found by CPI and YE, among others. It is more instructive to compare the rest-frame colors of the two samples. Using the spectral classifications from above, each galaxy's color was $k$-corrected, by interpolating in color between the closest typical galaxy sequences. We find that the mean rest-frame colors are nearly identical, with a very slight trend toward paired galaxies being redder. No apparent difference in the distribution of rest-frame colors is apparent from the histograms (Figure 8) or the K-S test. Therefore, we find no measurable differences between the $k$-corrected colors of paired and isolated galaxies. Mean rest-frame color differences of $|\Delta(g-r)| \gtrsim 0.1$ magnitudes can be ruled out at the $3 \sigma$ level.

\subsection{Luminosities}

The apparent magnitude distributions of the two samples are also very similar. In order to better compare luminosities, we calculate the absolute magnitude for each galaxy. Since we are only looking at relative luminosities, the results will not be sensitive to the 
choice of cosmological parameters. The $k$-correction in $r$ for each galaxy was extracted from the CNOC1 database. These $k$-corrections were derived using the data of Sebok (1986) for Gunn $r$, and depend only on the galaxy's redshift and color. The average $k$-correction for the paired sample $(0.27 \mathrm{mag})$ is identical to that for the isolated sample.

The resulting mean absolute magnitude of paired galaxies was found to be nearly identical to that of the isolated sample. This is confirmed with a K-S test (see Figure 9 also). A similar finding was made by YE. However, there is an indication that galaxies in

the paired (faint) sample are significantly more luminous than galaxies with no companions (isolated). This result is confirmed with a K-S test. The difference can be attributed to an inherent bias in selecting galaxies for the paired (faint) sample. Primary galaxies which are brighter in apparent magnitude will be more likely to have faint physical companions detected than will apparently fainter galaxies. This selection effect only comes into play when the apparent magnitude limit for companions is extended deeper than that for the primary sample, and hence does not affect the paired sample.

\section{5. $[\mathrm{OII}]$ Equivalent Widths}

One of the best indicators of current star formation is the presence of spectral emission lines such as [O II] $\lambda 3727$. Using a local sample of galaxies, Liu and Kennicutt (1995) found that the mean [O II] equivalent width was $19 \AA$ for merging galaxies, as compared to 11 $\AA$ for the complete sample. We used the procedure outlined in Abraham et al. (1996) to measure the $[\mathrm{O} \mathrm{II}]$ rest-frame equivalent widths of all the CNOC1 galaxies. We were able to determine equivalent widths for $81 \%$ of the primary sample. Typical errors are estimated to be $\lesssim 20 \%$. The mean [O II] equivalent width is found to be the same for paired and isolated galaxies. K-S tests and the visual appearance of the histograms (Figure 10) confirm this finding. CPI found a similar result. 


\subsection{Properties within Sub-samples}

The paired galaxy sample can be divided into two subsets : those which have at least one confirmed companion ("paired [phys]", based on velocity criterion of $\S$ 5.1), and those for which the companions have photometric information only ("paired [no z]"). All pairs in the former sample are physical (although not necessarily merging) and therefore would be expected to exhibit greater mean property differences from the isolated sample if merging affects galaxy properties significantly. The observed properties of these two samples (see Table 1) are found to be statistically equivalent to the isolated sample, strengthening the earlier conclusions. One might also ask how the properties of the companions themselves compare to the paired and isolated galaxies in the primary sample. Due to the nature of our sample selection (see Section 3), most of the companions which have redshifts are also part of the paired primary sample; therefore the mean properties are very similar. The mean apparent magnitude and color of the companions without redshifts are also found to be statistically equivalent to that of the isolated galaxies in the primary sample. To summarize, there are no observed differences in the overall properties of galaxies which have close companions of comparable luminosity and galaxies with no companions brighter than $r=23.0$.

\section{THE GALAXY MERGER RATE}

As mentioned earlier, the average redshift of the sample is 0.33 , which gives us some leverage in investigating the redshift dependence of the merger rate. For comparison, we will use a low redshift sample which is derived from the UGC catalog (Nilson 1973). We select all galaxies with measured diameters of at least $1^{\prime}$ in both $B$ and $R$, to a (statistically complete) limiting magnitude of $B=14.5$. Within this sample, the average redshift (for galaxies with measured redshifts) is 0.0076. At this distance, a physical separation of 20 
$h^{-1} \mathrm{kpc}$ corresponds to an angular separation of $184^{\prime \prime}$. We find that 130 out of 3058 galaxies have companions within this radius, yielding a close pair fraction of $4.3 \pm 0.4 \%$. Since optical companions are negligible at these bright magnitudes (see CPI), we take this to be the fraction of galaxies in close physical pairs. We note, however, that this estimate is approximate in nature. While the internal error in the local pair fraction is small, the systematic errors may be fairly large. A more complete redshift sample with well-defined selection effects is needed to improve this estimate.

Before comparing the two samples, we must ensure that the pair fraction does not depend strongly on the luminosities of the galaxies involved. For the nearby sample, $B$ $=14.5$ corresponds to an average absolute limiting magnitude of $M_{B}=-17.3$ (based on the average redshift). The CNOC1 primary field sample has an average apparent limiting magnitude of $r=21.3$, which corresponds to $\mathrm{B}=23.1$ using the Gunn color transformation of Windhorst et al.(1991) and the average color $(g-r=0.79)$ of the primary galaxies. The average $B k$-correction $\left(k_{B}=1.0\right)$ was estimated using the mean redshift $(z=0.33)$, the color classification fractions determined in Section 6.2, and the $k$-corrections of Frei \& Gunn (1994). This results in an average absolute limiting magnitude of $M_{B}=-18.1$, which is roughly one magnitude brighter than the low-redshift sample. It has been shown by YE that the UGC pair fraction remains basically unchanged over a range of $\sim 2$ mag brighter than $\mathrm{B}=14.5$; hence, we conclude that the two samples are of comparable luminosity, and the pair fractions derived should not be sensitive to the particular magnitude limits used.

We need to determine the fraction of galaxies in each sample which are likely to merge. While those in close physical pairs are likely candidates, some may be moving at such high relative velocities that they are unlikely to merge. Using dynamical arguments, CPI argue that, at the present epoch, pairs with relative velocities less than $350 \mathrm{~km} / \mathrm{s}$ are likely to merge. They estimate this fraction to be $\sim 50 \%$. For a pairwise velocity evolving as 
$(1+z)^{-1}$ (see CPI), this increases to a fraction of $66 \%$ at $z=0.33$. There are 13 close physical pairs of primary galaxies in our distant sample for which redshifts are available for both members. In this sample, 11 out of 13 pairs $(85 \pm 26 \%)$ have $\Delta V<350 \mathrm{~km} / \mathrm{s}$, which is consistent with the assumed pairwise velocity. Using the close pair fraction determined earlier in this section, we conclude that the local merging fraction is $2.1 \pm 0.2 \%$. It follows that, with $7.1 \pm 1.4 \%$ of our distant sample in close physical pairs, the merging fraction at $z=0.33$ is $4.7 \pm 0.9 \%$. If we assume the functional form of $(1+z)^{m}$ for the increase of the merger rate with redshift (Carlberg 1990), we find $\mathrm{m}=2.8 \pm 0.9$.

This value is consistent with the merger rate of CPI, who found $\mathrm{m}=3.4 \pm 1.0$ using the same method (and the same low redshift sample). We note, however, that they overestimated the local pair fraction by underestimating its mean redshift. After correcting for this, we find $\mathrm{m}=4.0 \pm 1.0$ for their sample. Our merger rate is significantly lower than that obtained using the physical pair fraction found by YE, which translates into $\mathrm{m}$ $\sim 5.0 \pm 1.5$ using our parameterization of the merger rate with redshift. Their sample is the largest pair redshift survey next to ours; hence, it is important to find out what is causing this discrepancy. One major difficulty with the YE redshift sample is that pairs are known to be under-selected. This effect was accounted for by randomly discarding redshifts in each two redshift pair, and then correcting for this. Their result was tested using the method outlined in $\S 4.2$. For the observed quantities, we use their original sample (before redshifts were discarded). Using $n p_{1}=7.2, n p_{0}=9.8, n_{0}=90, n n z=200$, and $N=376$, we find $\mathrm{S}=47 \%, \mathrm{R}=41 \%, \mathrm{P}=61 \%$, and $\mathrm{x}=16.3 \%$. The pair sampling $(\mathrm{R} / \mathrm{S})$ is 87 \%. YE estimate that $\sim 55 \%$ of their observed pairs are physical. This leads to a physical pair fraction of $8.9 \pm 3.9 \%$, which is considerably lower than the $15.5 \pm 6.6 \%$ reported in their study. This revised pair fraction leads to a merger rate of $\mathrm{m}=3.4 \pm 1.9$, which is consistent with our result. 
Using HST images, BKWF derived $\mathrm{m} \sim 2.5 \pm 0.5$, which would appear to agree with our merger rate. However, they parameterized the evolution of the merger rate in a different way (by taking the merger rate to be the $z$-derivative of the pair counts). If we use their pair fraction with our method, we find $\mathrm{m}=4.5 \pm 0.5$, which is significantly higher than our result. However, YE point out that the pair fraction of $34 \pm 9 \%$ used by BKWF is approximately twice as large as the physical pair fraction (due to optical pairs). After taking this into account, and using our parameterization of the merger rate evolution, we find $\mathrm{m} \sim 2.9$ for the BKWF sample, in agreement with our result.

\section{REDSHIFT-BINNED SAMPLES}

An additional test of the validity of the preceding results is to divide the sample into redshift bins. The idea here is to look for redshift dependence in various properties, and also to have independent samples to look for common trends. We wish to have redshift bins which are small enough to avoid properties varying with redshift and large enough to ensure good statistics. Hence, we designate the following two samples : $0.18<z<0.30$ and 0.30 $<z<0.45$. This spans all primary field galaxies with $z \leq 0.45$, and avoids the sparsely populated high redshift regime (see Figure 6). Both samples have $\sim 240$ field galaxies, allowing for good statistics. Note that both bins cover lookback time intervals of $\sim 0.68$ $h^{-1}$ Gyr (assuming $\Omega_{0}=1$ ), which each correspond to $\sim 10 \%$ of the age of the universe for

that model. The results of the following comparisons of various properties are summarized in Tables 2 and 3. 


\subsection{Galaxy Properties}

For each sample, there is no measurable difference between the observed or rest-frame colors of paired and isolated galaxies. This is consistent with the findings of YE, who used similar redshift bins. In the low redshift bin, paired galaxies are slightly more luminous on average, but the difference is at the one sigma level. The mean absolute magnitudes are statistically equivalent in the high redshift bin. No significant differences were found in the classifications of paired and isolated galaxies in either redshift bin (note that very high

K-S test significance levels are the result of the discrete classification systems). YE, on the other hand, found a larger emission line fraction in their high-redshift pair sample, but the difference was at the one sigma level.

\subsection{Pair Fractions}

As with the entire sample, we wish to investigate the pair fraction. However, by using the same apparent magnitude criteria as before, we encounter the problem that the low redshift sample will contain intrinsically fainter companions than the high redshift sample, rendering a direct comparison invalid. In order to compare the two redshift bins, we instead impose different limiting magnitudes, to sample galaxies of comparable luminosity. For this comparison, we relax the constraint that the secondary samples be drawn from populations of similar luminosity as the primary sample. For the high redshift bin, we impose the faintest magnitude limit possible $-r=23.0$. We can estimate the absolute magnitude of the faintest companions detectable in this bin by using $z=0.45$ and the average $k$-correction of $0.27 \mathrm{mag}$, yielding $M_{r}=-18.1$. For the low redshift bin, the average $k$-correction is 0.19 mag. In order that the faintest companions in the low redshift bin also have $M_{r}=-18.1$, it follows that we must impose an apparent magnitude limit of $r=22.0$. Since all primary galaxies are brighter than this, we can do this without reducing the size of the primary 
sample.

Using the two defined samples, the pair fractions were determined. The fraction of galaxies in close physical pairs for the low redshift bin is $10.2 \pm 3.2 \%$, as compared to 11.3 $\pm 3.5 \%$ for the high redshift bin. The pair fractions for the redshift binned samples are larger than the fraction derived earlier for the entire sample, but this is to be expected since fainter companions have been included. A more suitable comparison is the merging fraction, found to be $6.3 \pm 2.0 \%$ for the low redshift bin, as opposed to $7.6 \pm 2.4 \%$ for the high redshift bin. In principle, these two independent quantities can be used to measure the merger rate evolution; however, the small numbers of galaxies makes this measurement $(\mathrm{m}=2.1 \pm 5.2)$ nearly meaningless for our sample. However, larger redshift surveys will be able to place tighter constraints on the redshift dependence of the merger rate using this approach.

\section{PROPERTIES OF CLOSE PHYSICAL PAIRS}

In the following section, we will focus on close pairs in which both galaxies are members of the primary sample. In addition, we will concentrate only on those pairs in which the galaxies are physically associated (based on the relative velocity criterion outlined in $\S 5.1$ ). These pairs are the best candidates for systems undergoing mergers or interactions. Images of the 13 pairs satisfying these criteria are displayed in Figure 11 .

Close inspection of these images reveals clear evidence of ongoing interactions in some pairs. While the image quality is not sufficient to perform detailed morphological classifications of each galaxy, it is adequate to separate the pairs in the following three categories : interacting (int), possibly interacting (int?), or not interacting (no). Pair classification was carried out by DRP, CJP, and HKCY, with no prior knowledge of any 
other properties of the pairs or member galaxies. In all cases, at least two of us agreed on the classification, and there was no disagreement by more than one class. The majority classification was adopted in each case.

The pair identification number, rest-frame velocity difference, projected separation, and image classification for each pair is listed in Table 4. In addition, the galaxy identification number, rest-frame color, spectral classification, and [O II] rest-frame equivalent width is given for each galaxy. It is immediately apparent that the four pairs classified as interacting (int) are very blue, with exceptionally strong emission lines. In addition, all four pairs have low $(<150 \mathrm{~km} / \mathrm{s})$ relative velocities. Since each of these measures is determined independently, this suggests that these pairs are in the process of merging. Such strong evidence of merging is surprising in the light of earlier analysis on the mean properties of this sample. That is, while the mean properties of these galaxies are indistinguishable from the field, a subset of the pairs do exhibit significantly different properties. This shows that indications of mergers are being swamped by non-merging pairs in our sample of close pairs.

We clearly have not identified all galaxies in the sample which are currently affected by mergers. First of all, there are some merging pairs which would be missed by our approach. Galaxies with very close $\left(<1^{\prime \prime}\right)$ companions may appear as isolated galaxies on our images. Also, some galaxies may have close companions which fall below the magnitude limit imposed in our study. In both cases, even if these companions are relatively small, it is possible that minor mergers may trigger strong starburst activity (Mihos \& Hernquist 1994). Secondly, there are probably isolated galaxies in the field that have recently undergone mergers, and no longer have detectable companions nearby. These galaxies will obviously be missed in a study of close pairs. They may be identified as merger remnants if they have distorted appearances. However, as demonstrated by Mihos (1995), morphological signs of interactions are relatively short-lived, and can be difficult to detect at these redshifts. The 
presence of emission lines (such as $[\mathrm{O}$ II] $)$ can be used independently to detect on-going merger-induced starbursts, while other spectral indices (e.g. $H \delta$ ) serve as useful diagnostics of recent starburst activity (see Couch \& Sharples 1987). Furthermore, AGB light resulting from a starburst may last for several Gyr after a merger (Silva \& Bothun 1995). Finally, we note that some mergers may not produce any obvious signs that a merger is taking place. This is particularly true for mergers of early-type galaxies, which will not produce either a strong starburst or clear morphological signs of interaction.

\section{DISCUSSION}

Using a redshift-selected sample of field galaxies with $\langle z\rangle \sim 0.33$, we find that roughly $7 \%$ have close physical companions of comparable luminosity. This is a factor of 1.7 higher than the physical pair fraction found locally. The mean properties of these paired galaxies are found to be indistinguishable from those of isolated galaxies. Upon dividing the sample into two independent sets based on redshift, we find a similar result. The only trend we find, although not statistically significant, is for paired galaxies to be (on average) slightly redder, more luminous, and of earlier type-classification than galaxies with no close companions. These results are consistent with evidence that early-type galaxies are more clustered than late-type galaxies (Roberts \& Haynes 1994). For example, Loveday et al. (1995) find that early-type galaxies are clustered more strongly than late-type galaxies by a factor of 3.5 - 5.5 locally, while Neuschaefer et al. (1995) find a similar effect in a sample with an average redshift of $z \sim 0.5$.

Upon examining the images of the 13 pairs which are known to be physically associated (based on similar redshifts), we find that the galaxies in pairs which are classified as interacting (based on their appearances) do exhibit very different properties. These pairs all have low relative velocities, and their galaxies have very blue rest-frame colors and spectra 
with strong emission lines. This is the first clear evidence (at this redshift) that paired galaxies have significantly different properties than field galaxies in general. This implies that mergers are responsible for at least some of the starbursting activity seen in samples of faint field galaxies. There are two ways to improve on this result. First, a larger and more complete redshift sample will increase the yield of close physical pairs. Secondly, high resolution images would allow for improved detection of signs of interactions in these pairs.

We can use the information in Figure đb to make a rough estimate of the mean dynamical friction timescale for the close physical pairs in our sample. Following Binney \& Tremaine (1987), we can estimate the time needed for a companion to merge. Taking projected separations and relative velocities from Table 4, and assuming conservative values for the Coulomb logarithm $(\ln \Lambda \sim 3)$ and galaxy mass-to-light ratio $(M / L \sim 5)$, we find a mean timescale of $\sim 150$ Myr. Strictly speaking, this technique doesn't apply to mergers between galaxies of comparable mass (the typical mass ratio is 2:1 for these pairs); however, it confirms the assumption that the timescales involved are short. An alternative approach is to use the method outlined by Charlton and Salpeter (1991) for mergers between galaxies of comparable mass. Assuming the inward drift due to dynamical friction is independent of separation (once halos overlap appreciably), and adopting a typical number of decay orbits (3) based on N-body calculations, we find a mean dynamical friction timescale of $\sim$ 400 Myr. Again, while this method is approximate in nature, it indicates that these close physical pairs should merge quickly, as expected.

The merger rate is estimated to increase with redshift as $(1+z)^{m}$, with $\mathrm{m}=2.8 \pm 0.9$. We demonstrate that our determination of the increase in the merger rate with redshift is consistent with earlier determinations using close pairs, and provides one of the strongest constraints to date. The observed increase in the merger rate with redshift implies that the average galaxy mass at $z \sim 0.33$ was substantially lower. Our observed value of $\mathrm{m}$ is 
consistent with the theoretical value of $\mathrm{m}=2.5$, derived by Toomre (1977) using binding energies of bound pairs. Carlberg (1990) estimated that $\mathrm{m} \simeq 4.51 \Omega^{0.42}$, for $\Omega$ near 1 and a CDM-like cosmology. This implies that our result is consistent with a low density universe, as found in several recent studies (e.g. Carlberg et al. 1996). Our result is also consistent with merging being the cause of the observed evolution in co-moving luminosity density found by Lilly et al. (1996). For blue wavelengths, they find $L \propto(1+z)^{2.3 \pm 0.5}$ for $\Omega_{0}=0.1$ and $q_{0}=0.05\left(L \propto(1+z)^{2.7 \pm 0.5}\right.$ for $\Omega_{0}=1$ and $\left.q_{0}=0.5\right)$. They attribute this evolution to a rapid decline in the global star formation rate since $z \sim 1$.

There are several obvious ways of improving on the results of this paper. A larger and more complete set of redshifts would decrease the swamping effect of unrelated optical pairs, as well as simply reducing the uncertainty in estimating the merger rate and differences in various properties. Secondly, additional spectral features and colors may prove to be better diagnostics of on-going or recent mergers. Finally, a significant short-coming of this type of study is simply a lack of resolution. High resolution imaging would allow close faint companions to be identified, purifying the pair sample. In addition, improved morphological indications of ongoing mergers or disruptions would help to identify merger candidates. Quantitative determinations of morphology of individual galaxies would also allow the competing effects of mergers and the morphology-density relation to be disentangled more easily.

We wish to thank other members of the CNOC consortium for assistance in carrying out the observations and data reduction. We are grateful to Mike Balogh and Luc Simard for their assistance in measuring [O II] line strengths. We also wish to thank Mike Hudson, Ron Marzke, and Simon Morris for helpful discussions. This work was supported by the Natural Sciences and Engineering Research Council, through research grants to CJP, HKCY and RGC, and a post-graduate scholarship to DRP. 


\section{REFERENCES}

Abraham, R. G., Smecker-Hane, T. A., Hutchings, J. B., Carlberg, R. G., Yee, H. K. C., Ellingson, E., Morris, S., Oke, J. B., \& Rigler, M. 1996, ApJS, in press

Barnes, J. E. 1988, ApJ, 331, 699

Binney, J., \& Tremaine, S. 1987, in Galactic Dynamics (Princeton Princeton University Press)

Broadhurst, T. J., Ellis, R. S., \& Shanks, T. 1988, MNRAS, 235, 827

Broadhurst, T. J., Ellis, R. S., \& Glazebrook, K. 1992, Nature, 355, 55

Burkey, J. M., Keel, W. C., Windhorst, R. A., \& Franklin, B. E. 1994, ApJ, 429, L13 $(\mathrm{BKWF})$

Carlberg, R. G. 1990, ApJ, 359, L1

Carlberg, R. G., \& Charlot, S. 1992, ApJ, 397, 5

Carlberg, R. G., Pritchet, C. J., \& Infante, L. 1994, ApJ, 435, 540 (CPI)

Carlberg, R. G. 1995, in Galaxies in the Young Universe, ed. H. Hippelein, K. Meisenheimer, \& H.-J. Röser (Springer-Verlag, Berlin) p. 206.

Carlberg, R. G., Yee, H. K. C., Ellingson, E., Abraham, R., Gravel, P., Morris, S., \& Pritchet, C. J. 1996, ApJ, 462, 32

Charlton, J. C., \& Salpeter, E. E. 1991, ApJ, 375, 517

Colless, M., Ellis, R. S., Taylor, K., \& Hook, R. N. 1990, MNRAS, 244, 408

Colless, M., Ellis, R. S., Broadhurst, T. J., Taylor, K., \& Peterson, B. A. 1993, MNRAS, 261,19

Coleman, G. D., Wu, C. C., \& Weedman, D. W. 1980, ApJS, 43, 393 (CWW)

Couch, W. J., \& Sharples, R. M. 1987, MNRAS, 229, 423 
Driver, S. P., Windhorst, R. A., \& Griffiths, R. E. 1995, ApJ, 453, 48

Ellingson, E., \& Yee, H. K. C. 1994, ApJS, 92, 33

Frei, Z., \& Gunn, J. E. 1994, AJ, 108, 1476

Glazebrook, K., Ellis, R., Santiago, B., \& Griffiths, R. 1996, MNRAS, in press

Griffiths, R. E., et al. 1994, ApJ, 435, L19

Infante, L., \& Pritchet, C. J. 1995, ApJ, 439, 565

King, C., \& Ellis, R. S. 1985, ApJ, 288, 456

Koo, D. C., \& Kron, R. G. 1992, ARA\&A, 30, 613

Larson, R. B., \& Tinsley, B. M. 1978, ApJ, 219, 46

Lilly, S. J., Tresse, L., Hammer, F., Crampton, D., \& Le Fèvre, O. 1995, ApJ, 455, 108

Lilly, S. J., Le Fèvre, O. L., Hammer, F., \& Crampton, D. 1996, ApJ, 460, L1

Lin, H., Yee, H. K. C., Carlberg, R. G., \& Ellingson, E. 1996, ApJ, in press

Liu, C. T., \& Kennicutt, R. C. 1995, ApJ, 450, 547

Loveday, J., Maddox, S. J., Efstathiou, G., \& Peterson, B. A. 1995, ApJ, 442, 457

Mihos, J. C., \& Hernquist, L. 1994, ApJ, 425, L13

Mihos, J.C. 1995, ApJ, 438, L75

Nilson, P. 1973, Uppsala General Catalog of Galaxies (Uppsala: Royal Society of Sciences of Uppsala)

Neuschaefer, L.W., Ratnatunga, K. U., Griffiths, R. E., Casertano, S., \& Im, M. 1995, ApJ, 453,559

Peebles, P. J. E. 1980, in The Large Scale Structure of the Universe (Princeton : Princeton University) 
Roberts, M. S., \& Haynes, M. P. 1994, ARA\&A, 32, 115

Rocca-Volmerange, B., \& Guiderdoni, B. 1990, MNRAS, 247, 166

Sebok, W.L. 1986, ApJS, 62, 301

Silva, D. R., \& Bothun, G. D. 1995, BAAS, 187, 83.07

Toomre, A. 1977, in Evolution of Galaxies and Stellar Populations, ed. B.M. Tinsley, \& R.B. Larson (New Haven:Yale Obs.), p. 401

Tyson, J. A. 1988, AJ, 96, 1

Windhorst, R. A., et al. 1991, ApJ, 380, 362

Woods, D., Fahlman, G. G., \& Richer, H. B. 1995, ApJ, 454, 32

Yee, H. K. C., \& Ellingson, E. 1995, ApJ, 445, 37 (YE)

Yee, H. K. C., Ellingson, E., \& Carlberg, R. G. 1996, ApJS, 102, 269 (YEC)

Zepf, S. E., \& Koo, D. C. 1989, ApJ, 337, 34 


\section{FIGURE CAPTIONS}

Fig. 1. - The selection function for all galaxies in the region of the cluster E1621. The lines represent the restrictions imposed to ensure at least $25 \%$ spectroscopic completeness for each galaxy in the primary sample.

Fig. 2.- Histogram of the local number density of galaxies within a $1^{\prime}$ radius of each unbiased field galaxy, using a limiting magnitude of $r=22.0$.

Fig. 3.- Histogram of the pair number density, binned in annular rings of width $0 . " 5$. The lines represents the pair number density predicted using the $F$ band angular correlation function from Infante \& Pritchet (1995), with $\delta=0.8$ (solid line) and $\delta=0.6$ (dotted line). Note the absence of pairs at very small angular separations, due to the limited resolution of the observations. (a) All galaxies brighter than $r=23.0$. (b) All galaxies brighter than $r=$ 23.0, excluding known cluster members.

Fig. 4.- Projected separation (D) versus rest-frame velocity difference $(\Delta V)$ for galaxy pairs with 2 redshifts. Points with small projected separations and small velocity differences represent pairs which are physically associated. $\Omega_{0}=1$ was assumed for this calculation. $(a)$ Pairs with two redshifts satisfying $\Delta V<10000 \mathrm{~km} / \mathrm{s}$ and $\mathrm{D}<100 \mathrm{~h}^{-1} \mathrm{kpc}$. (b) The subset of close physical pairs of primary galaxies.

Fig. 5. - The determination of a color cutoff. For a given primary galaxy at redshift $z$, all companions without redshifts which lie more than 0.15 magnitudes redward of the E/S0 sequence are assumed to lie at larger redshift. Hence, any such companions are expected to be unrelated. The non-evolving E/S0 sequence (solid line) was determined by convolving the filters with a typical E/S0 galaxy spectrum from CWW. The dashed line lies 0.15 magnitudes redward of the E/S0 sequence. Filled symbols represent field galaxies from the primary sample; open symbols represent field galaxies which are not part of the primary sample. 
Fig. 6.- Histograms of redshifts for the paired and isolated samples. Paired galaxies have at least one companion of comparable luminosity. Paired (faint) galaxies have at least one companion brighter than $r=23.0$, but none of comparable luminosity. Isolated galaxies have no companions brighter than $r=23.0$.

Fig. 7.- The color classification of galaxies in the primary sample. Classification was determined based on the observed $g-r$ colors and redshifts. The lines represent the colors of non-evolving galaxies derived by YEC by convolving Gunn filter responses with the standard typical galaxy spectra of CWW. The different galaxy sequences and corresponding classifications are: E (solid line, open circles), Sbc (dotted line, filled circles), Scd (dashed line, open squares), and Im (long-dashed line, stars).

Fig. 8. - Histograms of rest-frame $g-r$ colors for the paired and isolated samples. Restframe colors were derived using $g-r k$-corrections from YEC (using spectra of CWW). Paired galaxies have at least one companion of comparable luminosity. Paired (faint) galaxies have at least one companion brighter than $r=23.0$, but none of comparable luminosity. Isolated galaxies have no companions brighter than $r=23.0$.

Fig. 9.- Histograms of absolute $r$ magnitudes for the paired and isolated samples. Determination of $\mathrm{M}_{r}$ uses $r$ band $k$-corrections derived from Sebok (1986) by YEC, and assumes $h=1$. Paired galaxies have at least one companion of comparable luminosity. Paired (faint) galaxies have at least one companion brighter than $r=23.0$, but none of comparable luminosity. Isolated galaxies have no companions brighter than $r=23.0$.

Fig. 10.- Histograms of $[\mathrm{OII}]$ rest-frame equivalent widths for the paired and isolated samples. Paired galaxies have at least one companion of comparable luminosity. Paired (faint) galaxies have at least one companion brighter than $r=23.0$, but none of comparable luminosity. Isolated galaxies have no companions brighter than $r=23.0$. 
Fig. 11. - Mosaic of images for 12 close physical pairs in which both galaxies are primary field galaxies. North is up and east is to the left. Each image is 40 pixels square, corresponding to $\sim 12$ ".5 on a side. Pair identifications correspond to those found in Table 4. The physical projected pair separation ranges from $4 \mathrm{~h}^{-1} \mathrm{kpc}(\mathrm{p} 06)$ to $19 \mathrm{~h}^{-1} \mathrm{kpc}$ (p11). The typical FWHM is $\sim 1$ ". 0 , and is fairly constant from image to image. 


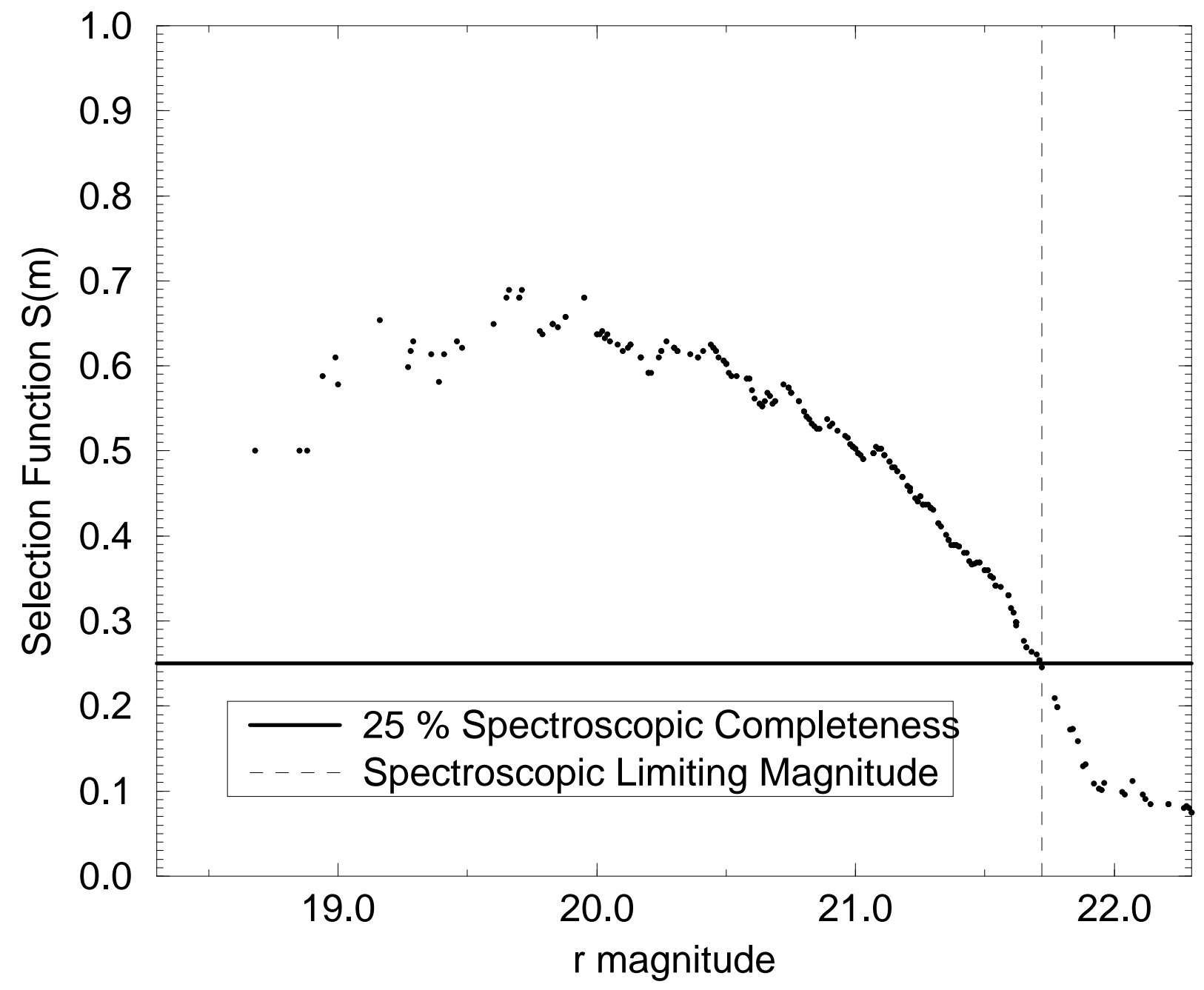




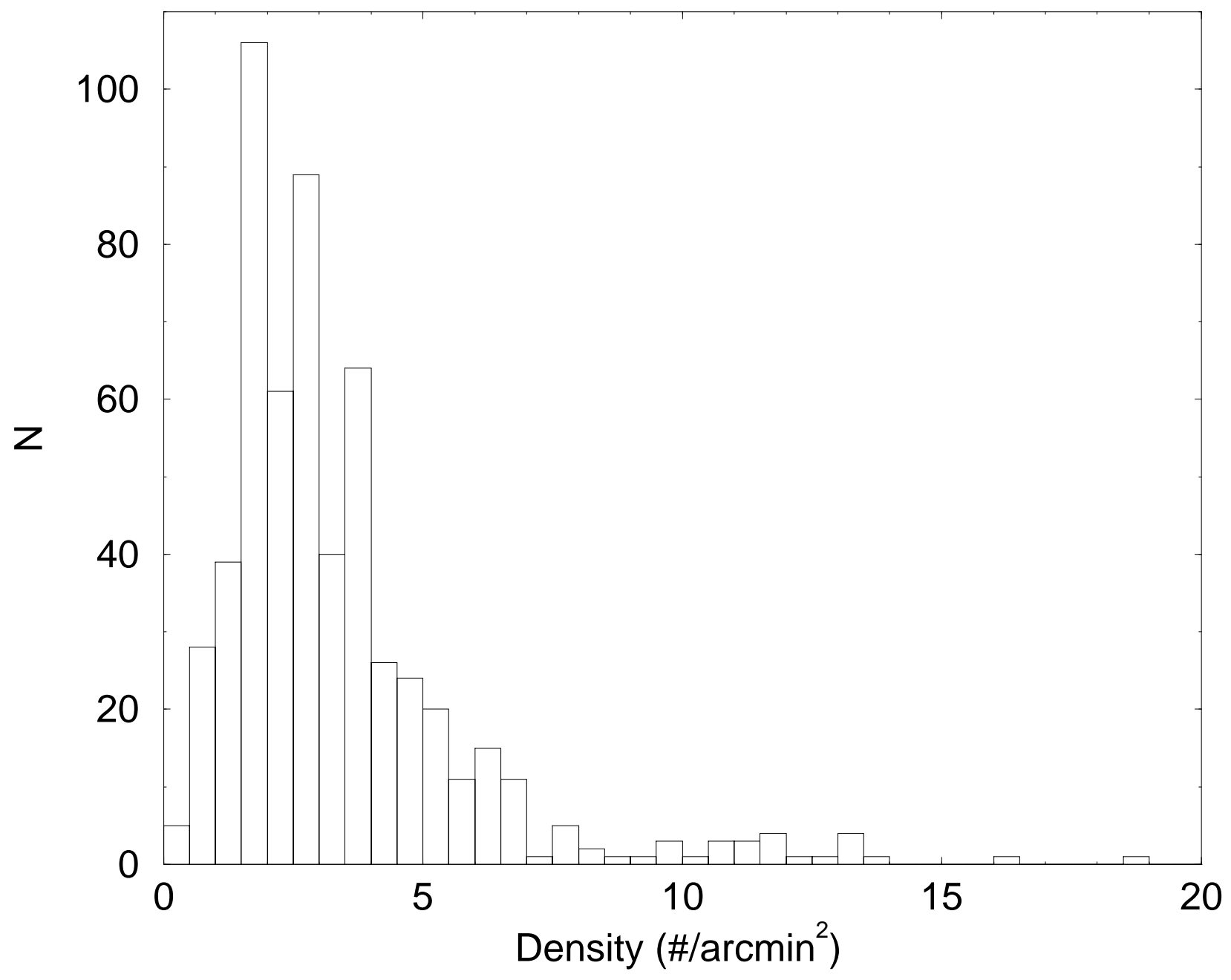



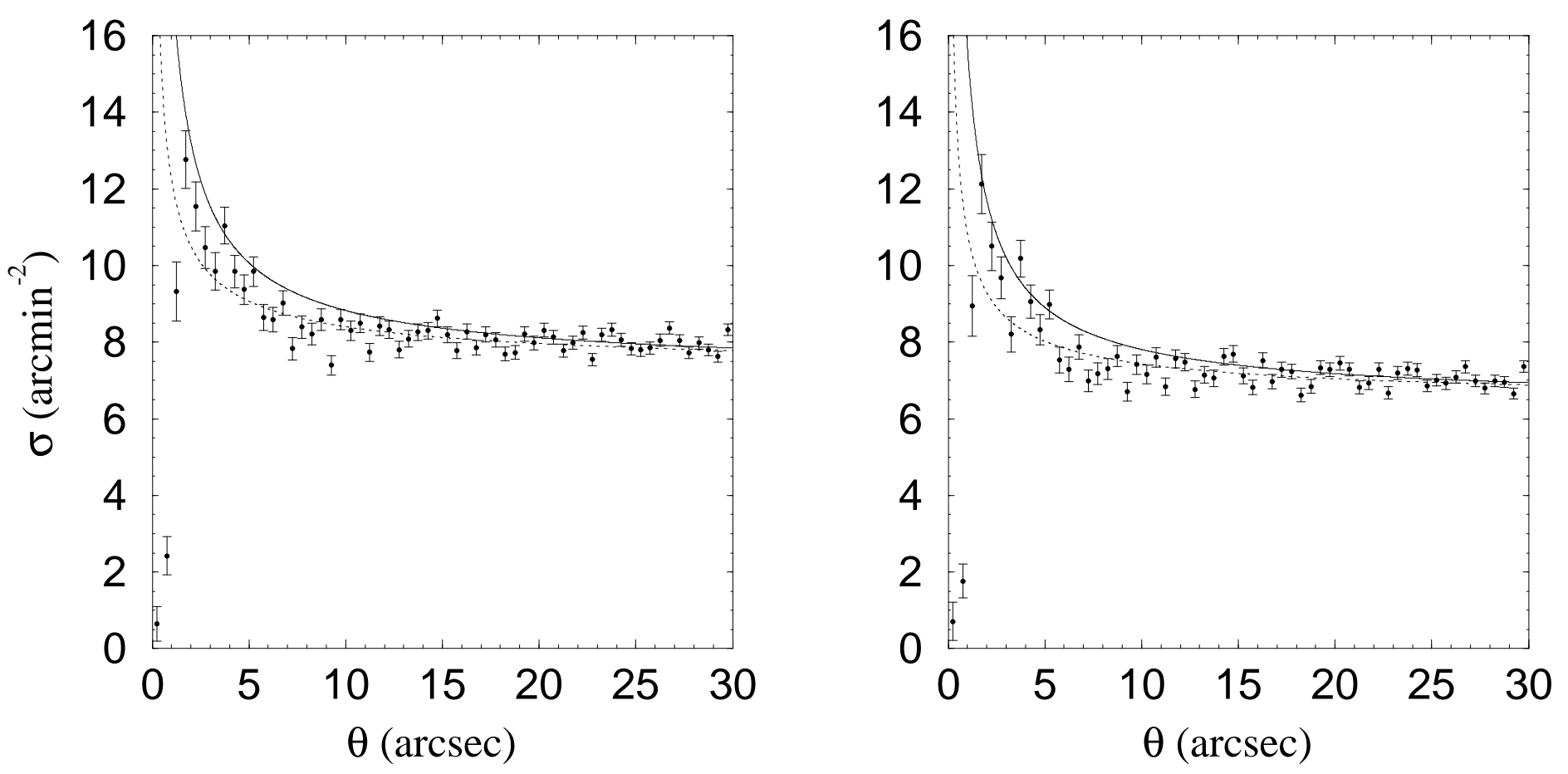

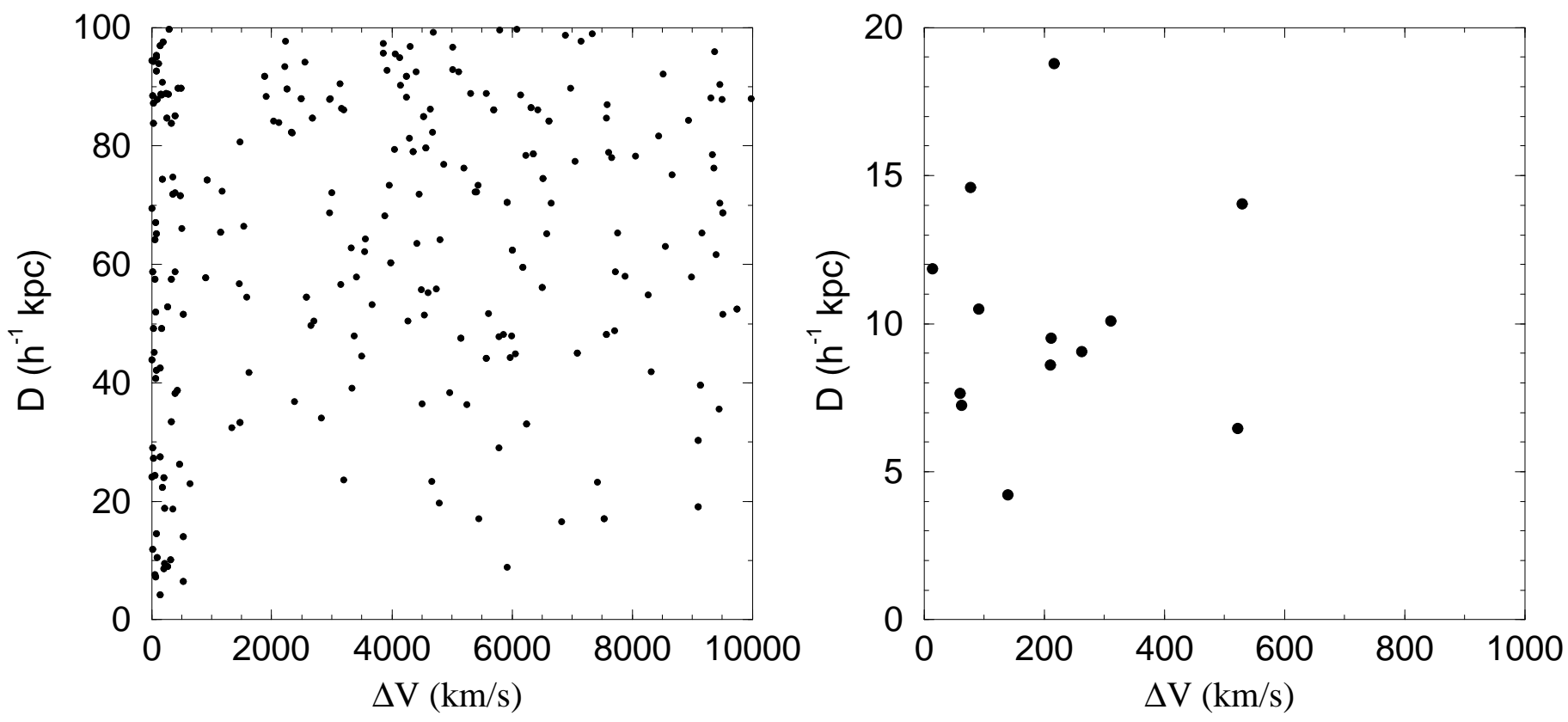


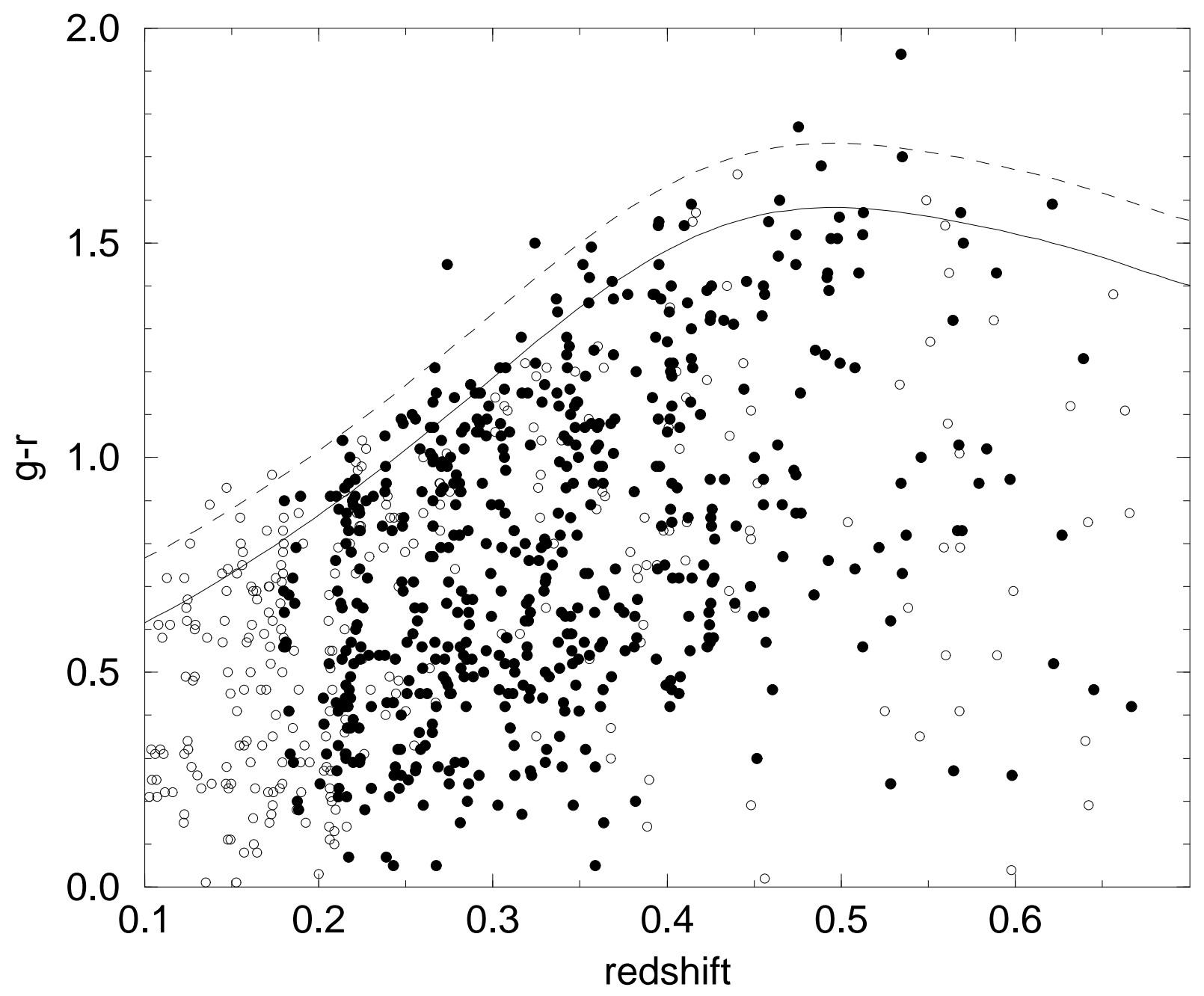



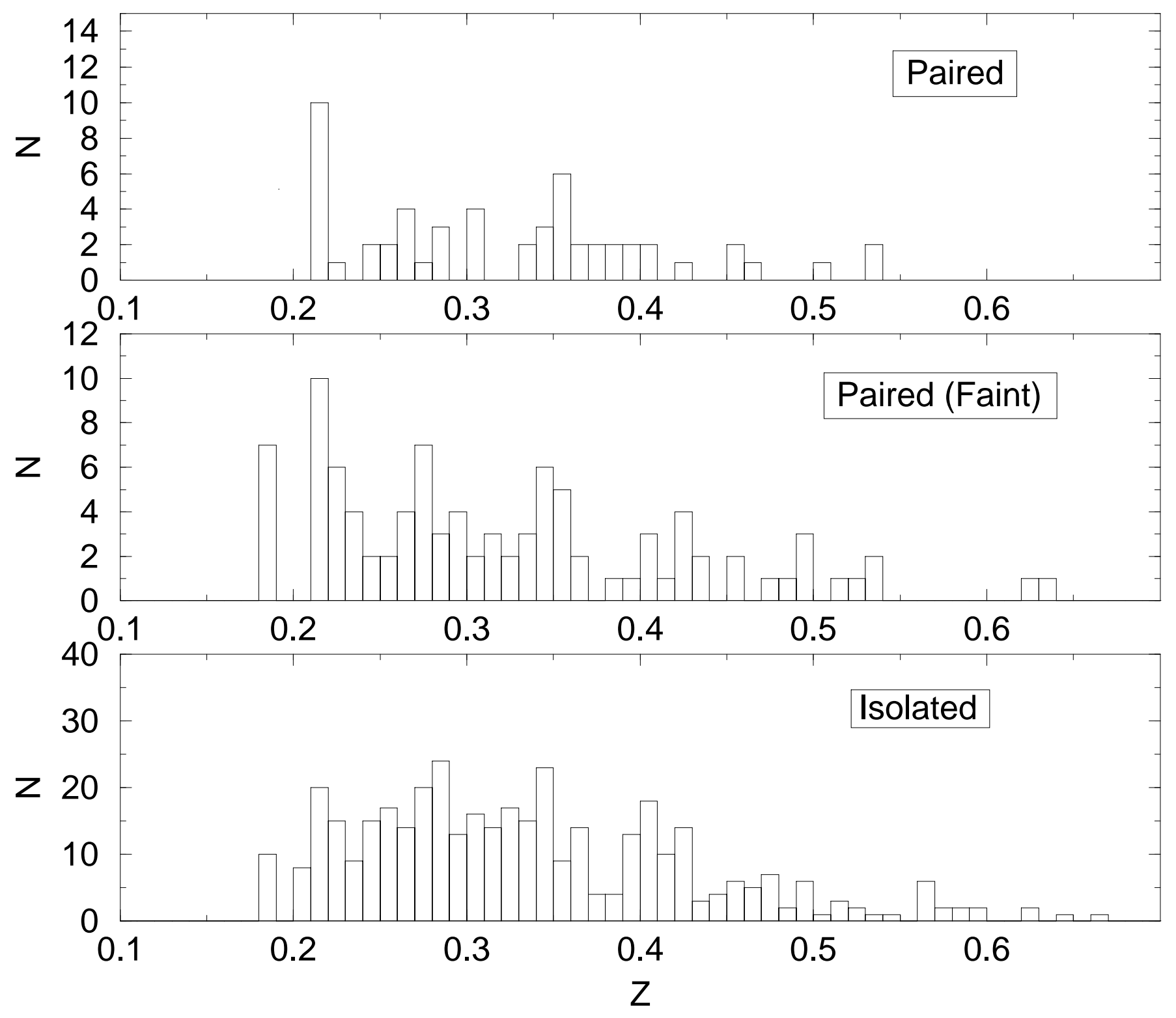


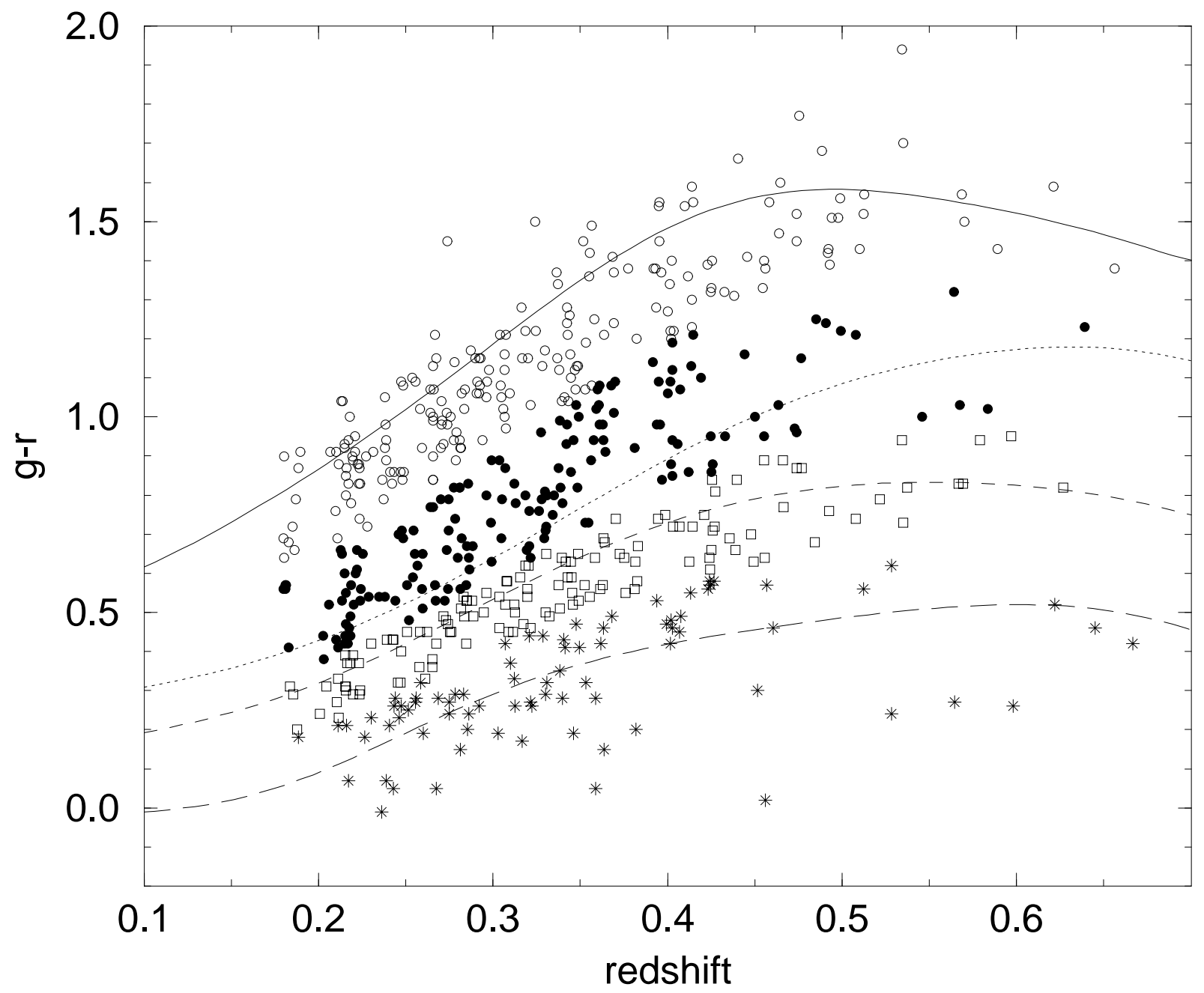



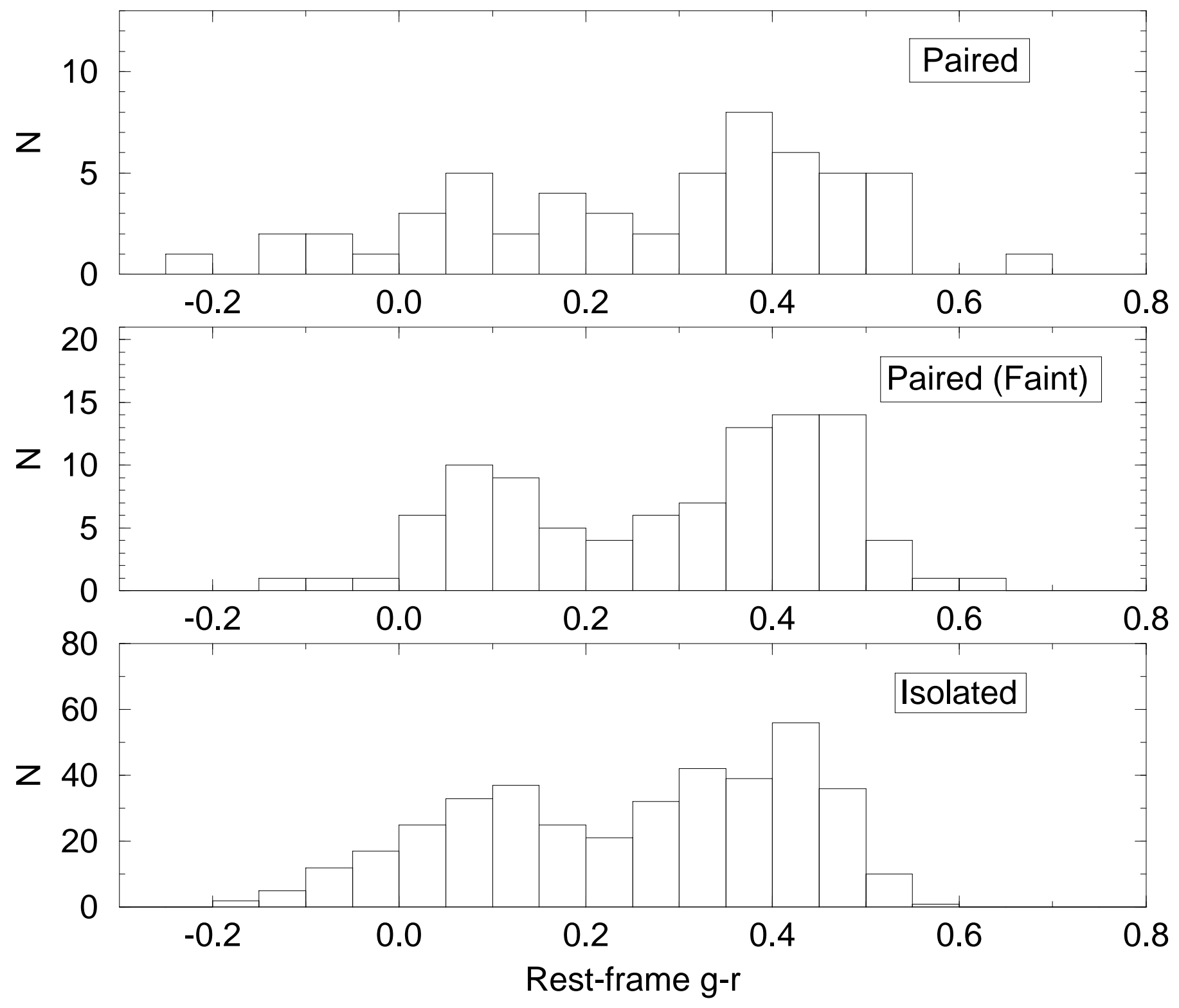

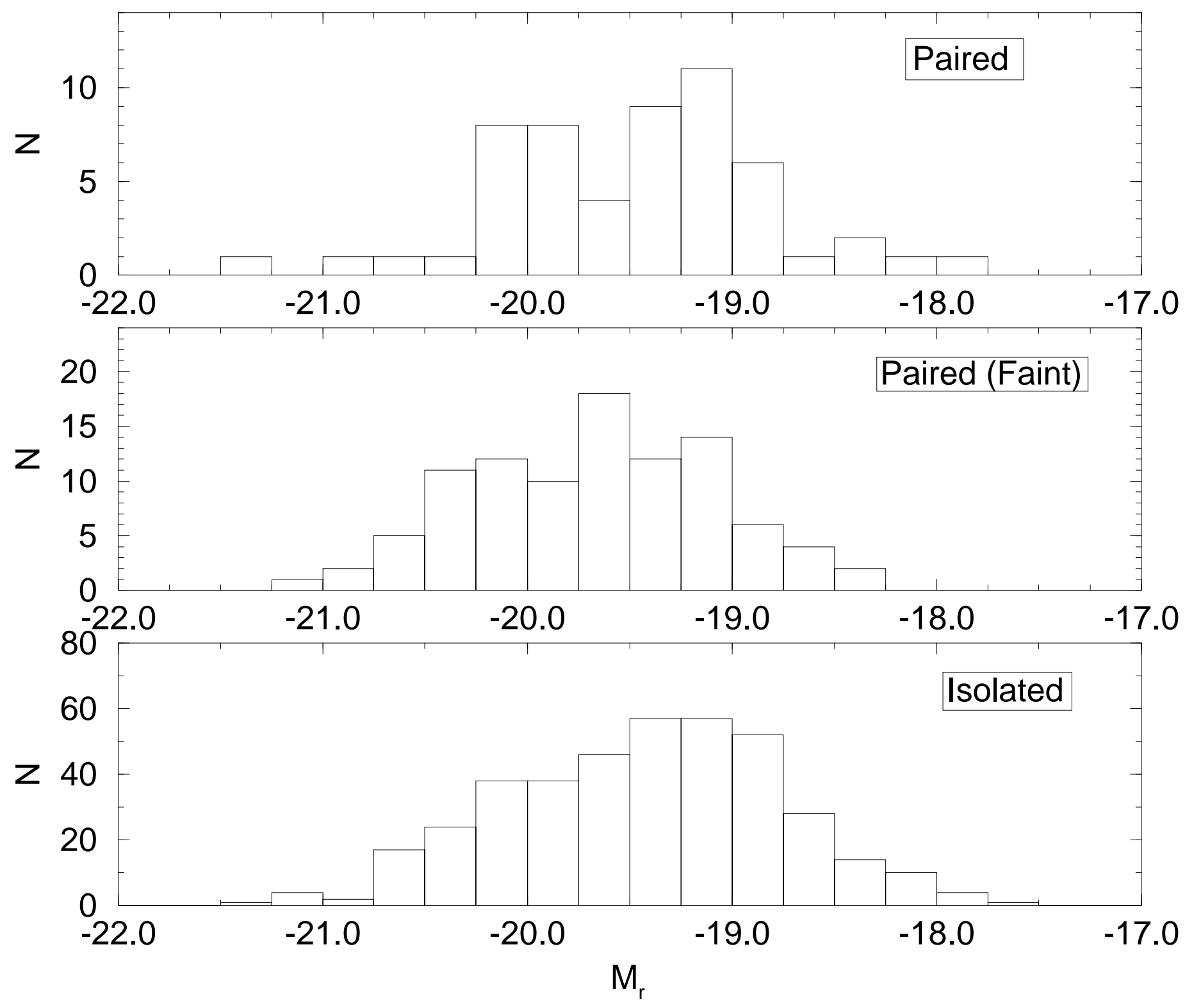

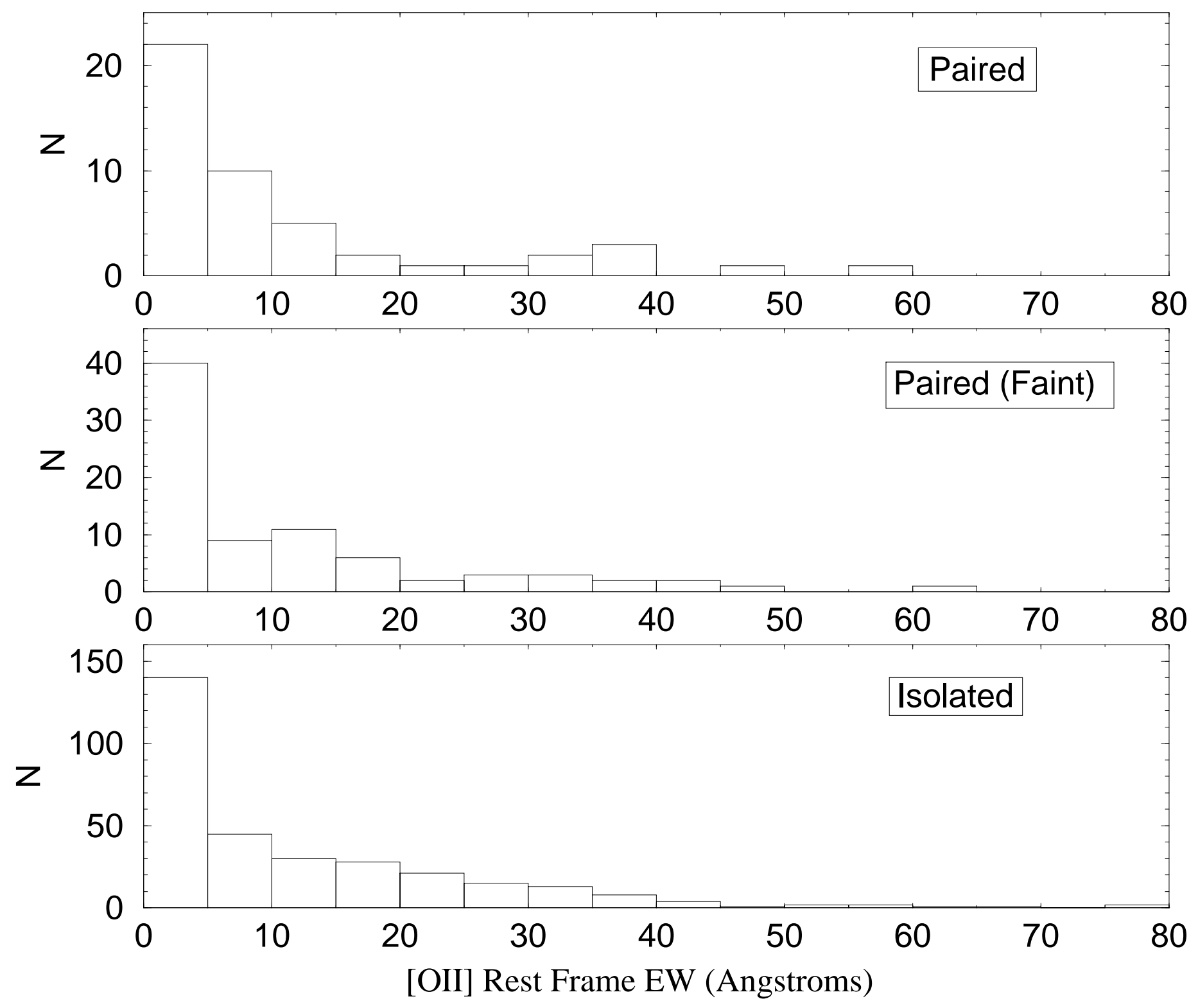
p01

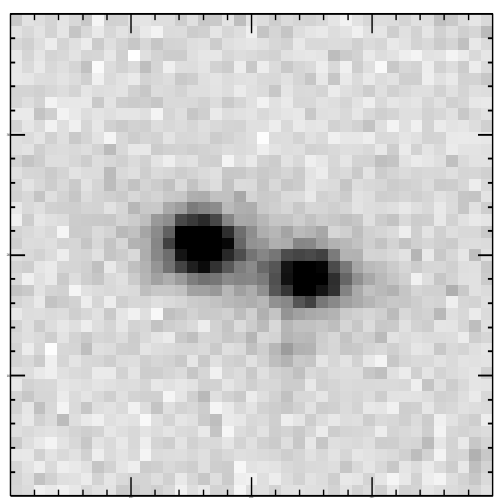

p04

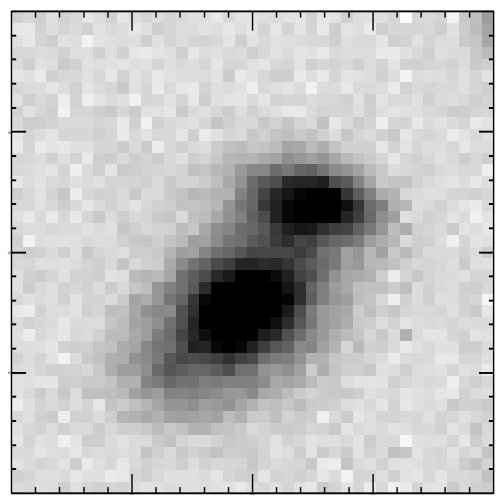

p07

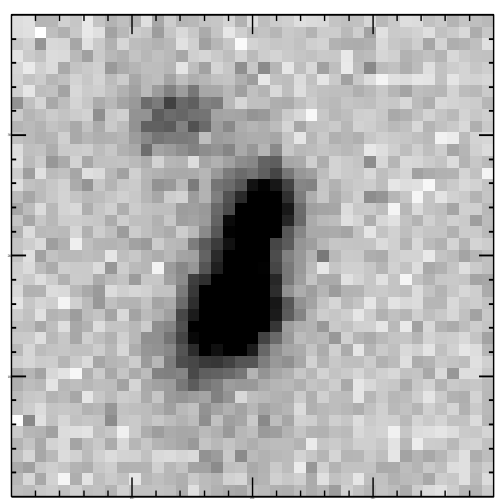

p10

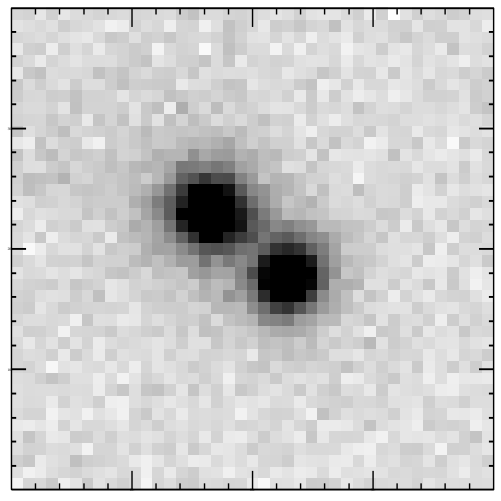

p02

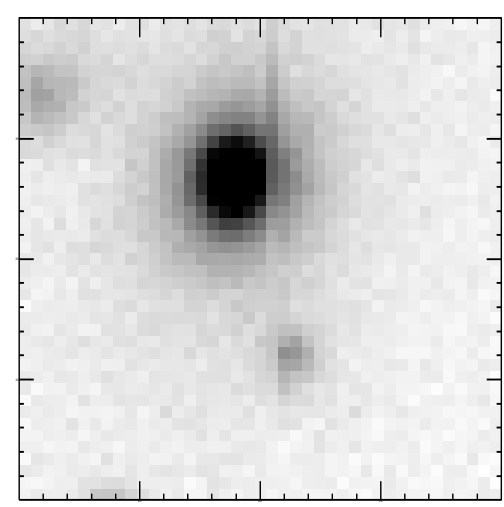

p05

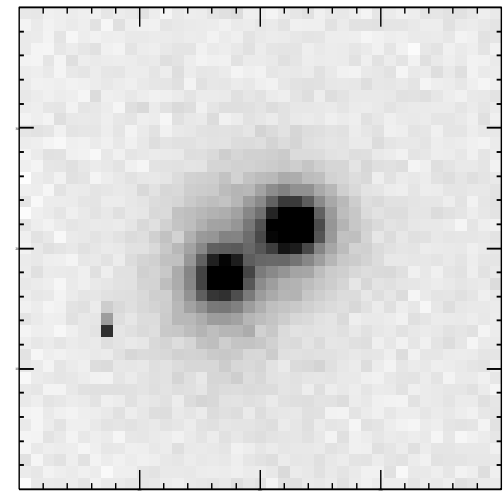

p08

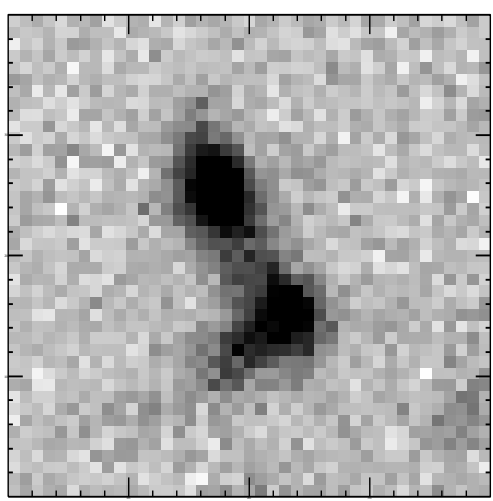

p11

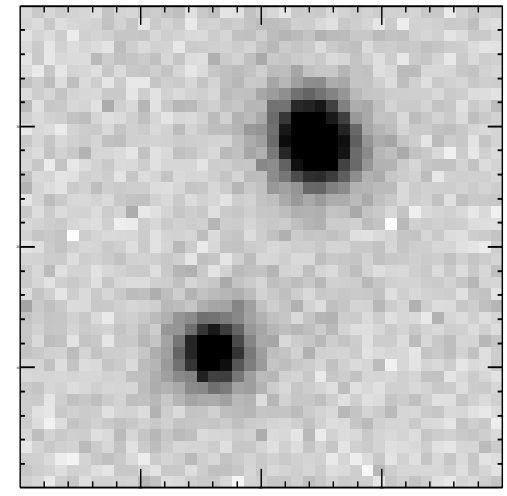

p03
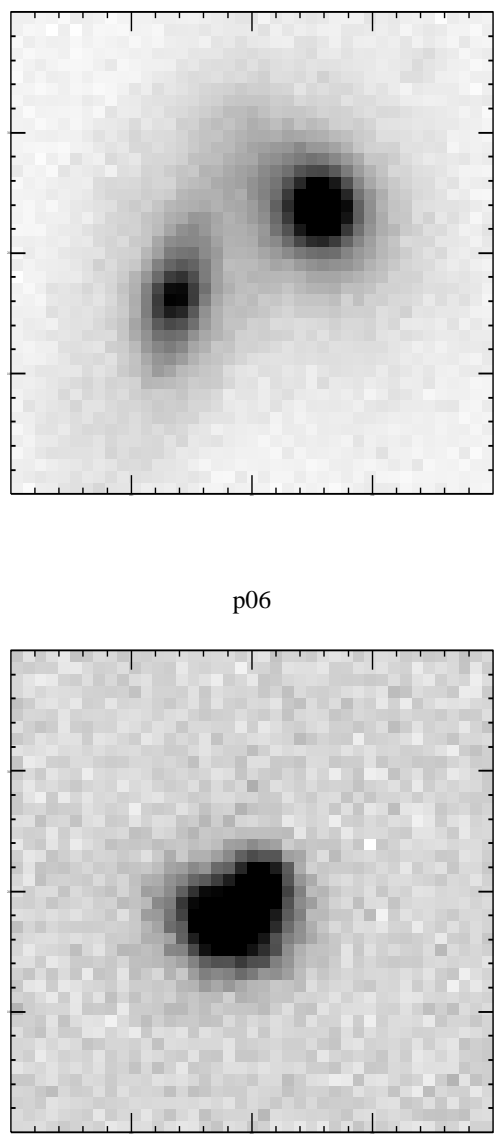

p09

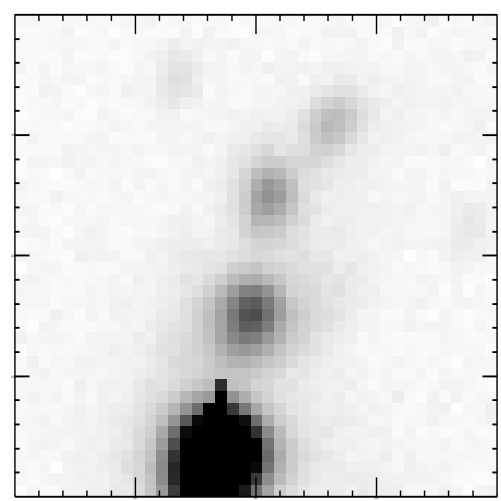

p12

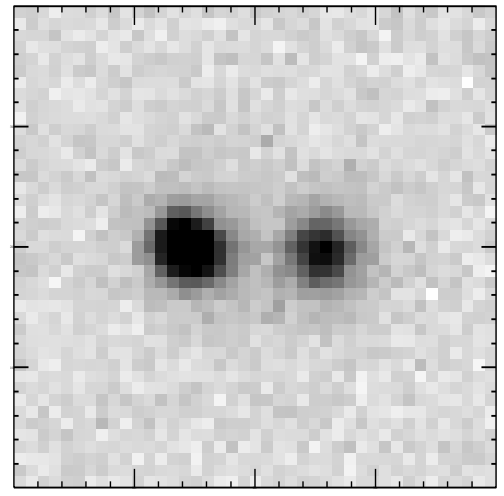



TABLE 1

Average Properties of Paired and Isolated Galaxies.

\begin{tabular}{|c|c|c|c|c|c|c|c|c|c|}
\hline $\begin{array}{l}\text { Galaxy } \\
\text { Sample }\end{array}$ & $\mathrm{N}$ & $\langle z\rangle$ & $\begin{array}{l}\text { Observed } \\
\langle g-r\rangle\end{array}$ & $\begin{array}{l}\text { Rest-frame } \\
\langle g-r\rangle^{\mathbf{a}}\end{array}$ & $\langle r\rangle$ & $<M_{r}>^{\mathrm{b}}$ & $E m^{c}$ & $S c d+I m{ }^{d}$ & 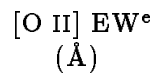 \\
\hline Paired & 55 & $0.325 \pm 0.012$ & $0.80 \pm 0.06$ & $0.26 \pm 0.03$ & $20.28 \pm 0.12$ & $-19.47 \pm 0.09$ & $29.1 \pm 7.3 \%$ & $36.4 \pm 8.1 \%$ & $10.9 \pm 2.0$ \\
\hline Paired (faint) & 97 & $0.322 \pm 0.011$ & $0.83 \pm 0.04$ & $0.29 \pm 0.02$ & $20.00 \pm 0.08$ & $-19.68 \pm 0.06$ & $25.8 \pm 5.2 \%$ & $33.0 \pm 5.8 \%$ & $10.5 \pm 1.5$ \\
\hline Isolated & 393 & $0.335 \pm 0.005$ & $0.78 \pm 0.02$ & $0.25 \pm 0.01$ & $20.37 \pm 0.04$ & $-19.43 \pm 0.03$ & $32.3 \pm 2.9 \%$ & $39.2 \pm 3.2 \%$ & $11.6 \pm \mathbf{0 . 8}$ \\
\hline Paired (phys) & 27 & $0.344 \pm 0.017$ & $0.96 \pm 0.10$ & $0.31 \pm 0.05$ & $20.47 \pm 0.19$ & $-19.36 \pm 0.15$ & $22.2 \pm 9.1 \%$ & $29.6 \pm 10.5 \%$ & $8.5 \pm 2.5$ \\
\hline Paired (no z) & 28 & $0.306 \pm 0.015$ & $0.65 \pm 0.06$ & $0.22 \pm 0.03$ & $20.09 \pm 0.15$ & $-19.58 \pm 0.10$ & $35.7 \pm 11.3 \%$ & $42.9 \pm 12.4 \%$ & $13.0 \pm 3.1$ \\
\hline Comp. (phys) & 27 & $0.344 \pm 0.017$ & $0.98 \pm 0.09$ & $0.33 \pm 0.04$ & $20.41 \pm 0.19$ & $-19.73 \pm 0.15$ & $18.5 \pm 8.3 \%$ & $25.9 \pm 9.8 \%$ & $8.5 \pm 2.5$ \\
\hline Comp. (no z) & 32 & & $0.73 \pm 0.06$ & & $20.54 \pm 0.15$ & & & & \\
\hline
\end{tabular}

${ }^{a}$ Using $g-r k$-corrections from YEC (using spectra of CWW).

${ }^{\mathrm{b}}$ Using $r k$-corrections derived from Sebok 1986 by YEC. Assumes $h=1$.

${ }^{c}$ Fraction of galaxies which have emission lines. Morphological types are determined by spectral classification. Errors assume Poisson statistics.

dFraction of galaxies which are Scd or Im. Morphological types are determined by color classification. Errors assume Poisson statistics.

${ }^{e}$ Approximately $90 \%$ of galaxies have equivalent widths measured. 
TABLE 2

K-S Test Significance Levels a for Paired/Isolated Galaxy Properties.

\begin{tabular}{|c|c|c|c|c|c|c|c|c|c|c|}
\hline $\begin{array}{c}\text { Redshift } \\
\text { Range }\end{array}$ & $\begin{array}{c}\mathrm{N} \\
\text { (Paired) }\end{array}$ & $\underset{\text { (Isolatedd) }}{\mathrm{N}}$ & $z$ & $\begin{array}{c}\text { Observed } \\
g-r\end{array}$ & $\begin{array}{c}\text { Rest-frame } \\
g-r\end{array}$ & $r$ & $M_{r}$ & $\begin{array}{l}\text { Spectral } \\
\text { Type }\end{array}$ & $\begin{array}{c}\text { Hubble } \\
\text { Type }\end{array}$ & 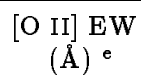 \\
\hline $0.18-0.67$ & $55^{\mathrm{b}}$ & 393 & 67 & 57 & 65 & 83 & 77 & $>99$ & $>99$ & 77 \\
\hline $0.18-0.67$ & $97^{c}$ & 393 & 15 & 79 & 13 & 0.00 & 0.38 & 89 & 36 & 76 \\
\hline $0.18-0.30$ & $23^{b}$ & 165 & 27 & 88 & 78 & 38 & 26 & $>99$ & 93 & 75 \\
\hline $0.30-0.45$ & $26^{\mathrm{b}}$ & 178 & 37 & 49 & 31 & $>99$ & 97 & 98 & 83 & 35 \\
\hline
\end{tabular}

Values given indicate the significance level (in percent) for the hypothesis that the two sets of data are drawn from the same distribution. Small values indicate that the two distributions have significantly different cumulative distribution functions.

${ }^{b}$ Field galaxies with companions of comparable luminosity.

cField galaxies with faint companions only.

dField galaxies with no companions brighter than $r=23.0$.

${ }^{e}$ Approximately $90 \%$ of galaxies have equivalent widths measured. 
TABLE 3

Average Properties in Redshift-Binned Samples.

\begin{tabular}{|c|c|c|c|c|c|c|c|c|c|}
\hline $\begin{array}{c}\text { Redshift } \\
\text { Range }\end{array}$ & $\begin{array}{l}\text { Galaxy } \\
\text { Sample }\end{array}$ & $\mathrm{N}$ & $\begin{array}{l}\text { Observed } \\
\langle\boldsymbol{g}-r\rangle\end{array}$ & $\begin{array}{l}\text { Rest-frame } \\
\langle\boldsymbol{g}-r\rangle^{\mathbf{a}}\end{array}$ & $\langle r\rangle$ & $<M_{r}>^{\mathbf{b}}$ & $E_{m}^{c}$ & $S c d+I m^{d}$ & {$\left[\begin{array}{c}\mathrm{O} \\
(\AA)\end{array}\right.$} \\
\hline $0.18-0.30$ & Paired & 23 & $0.59 \pm 0.06$ & $0.26 \pm 0.04$ & $19.74 \pm 0.18$ & $-19.49 \pm 0.16$ & $30.4 \pm 11.5 \%$ & $34.8 \pm 12.3 \%$ & $11.1 \pm \mathbf{3 . 2}$ \\
\hline $0.18-0.30$ & Paired (faint) & 49 & $0.67 \pm 0.04$ & $0.30 \pm 0.03$ & $19.58 \pm 0.09$ & $-19.60 \pm 0.09$ & $14.3 \pm 5.4 \%$ & $24.5 \pm 7.1 \%$ & $10.1 \pm 2.0$ \\
\hline $0.18-0.30$ & Isolated & 165 & $0.64 \pm 0.02$ & $0.28 \pm 0.01$ & $19.97 \pm 0.06$ & $-19.30 \pm 0.06$ & $26.7 \pm 4.0 \%$ & $34.5 \pm 4.6 \%$ & $10.9 \pm 1.4$ \\
\hline $0.30-0.45$ & Paired & 26 & $0.87 \pm 0.08$ & $0.24 \pm 0.04$ & $20.53 \pm 0.15$ & $-19.53 \pm 0.13$ & $23.1 \pm 9.4 \%$ & $38.5 \pm 12.2 \%$ & $10.6 \pm 3.0$ \\
\hline $0.30-0.45$ & Paired (faint) & 35 & $0.96 \pm 0.06$ & $0.28 \pm 0.03$ & $20.27 \pm 0.11$ & $-19.75 \pm 0.09$ & $34.3 \pm 9.9 \%$ & $37.1 \pm 10.3 \%$ & $11.2 \pm 2.8$ \\
\hline $0.30-0.45$ & Isolated & 178 & $0.83 \pm 0.02$ & $0.23 \pm 0.01$ & $20.53 \pm 0.05$ & $-19.54 \pm 0.05$ & $33.1 \pm 4.3 \%$ & $43.3 \pm 4.9 \%$ & $12.9 \pm 1.2$ \\
\hline
\end{tabular}

a Using $g-r k$-corrections from YEC (using spectra of CWW)

${ }^{\mathrm{b}}$ Using $r k$-corrections derived from Sebok 1986 by YEC. Assumes $h=1$

' Fraction of galaxies which have emission lines. Morphological types are determined by spectral classification. Errors assume Poisson statistics.

${ }^{\mathrm{d}}$ Fraction of galaxies which are Scd or Im. Morphological types are determined by color classification. Errors assume Poisson statistics.

${ }^{\mathrm{e}}$ Approximately $80 \%$ of primary galaxies have equivalent widths measured. 
TABLE 4

Properties of Close Physical Pairs.

\begin{tabular}{|c|c|c|c|c|c|c|c|c|}
\hline $\begin{array}{l}\text { Pair } \\
\text { ID }\end{array}$ & $\langle z\rangle$ & $\begin{array}{c}\Delta V \\
(\mathrm{~km} / \mathrm{s})\end{array}$ & $\begin{array}{c}\text { Separation } \\
\left(h^{-1} \mathrm{kpc}\right)\end{array}$ & $\begin{array}{l}\text { Image } \\
\text { Class. }^{a}\end{array}$ & $\begin{array}{c}\text { CNOC1 } \\
\text { Galaxy ID }\end{array}$ & $\begin{array}{c}\text { Rest-frame } \\
g-r^{\mathrm{b}}\end{array}$ & $\begin{array}{l}\text { Spectral } \\
\text { Class }^{c}\end{array}$ & $\begin{array}{c}{\left[\begin{array}{l}\mathrm{O} \\
\mathrm{II}\end{array}\right] \mathrm{EW}} \\
(\AA)\end{array}$ \\
\hline \multirow[t]{2}{*}{ p01 } & 0.46 & 91 & 10.5 & int? & E0015: 1560 & 0.41 & Em? & $<5$ \\
\hline & & & & & E0015: 1558 & 0.41 & Em & 12.3 \\
\hline \multirow[t]{2}{*}{ p02 } & 0.31 & 530 & 14.0 & no & E0302: 741 & 0.46 & no & $<5$ \\
\hline & & & & & E0302: 726 & 0.42 & no & 8.9 \\
\hline \multirow[t]{2}{*}{ p03 } & 0.22 & 311 & 10.1 & int? & E0839: 130289 & 0.51 & no & $<5$ \\
\hline & & & & & E0839: 130282 & 0.47 & no & 11.9 \\
\hline \multirow[t]{2}{*}{ p04 } & 0.22 & 61 & 7.7 & int & E0839: 130451 & 0.31 & Em? & 5.3 \\
\hline & & & & & E0839: 130460 & 0.09 & Em & 13.1 \\
\hline \multirow[t]{2}{*}{ p05 } & 0.35 & 522 & 6.5 & int? & E1224: 1635 & 0.47 & no & $<5$ \\
\hline & & & & & E1224: 1632 & 0.51 & no & $<5$ \\
\hline \multirow{2}{*}{ p06 } & 0.38 & 140 & 4.2 & int & E1512: 202223 & 0.11 & Em? & 13.4 \\
\hline & & & & & E1512: 202227 & -0.13 & Em? & 13.8 \\
\hline \multirow[t]{2}{*}{ p07 } & 0.34 & 63 & 7.2 & int & E1512: 200400 & -0.07 & Em? & 8.4 \\
\hline & & & & & E1512: 200408 & 0.15 & $\mathrm{Em}$ & 35.3 \\
\hline \multirow[t]{2}{*}{ p08 } & 0.36 & 15 & 11.8 & int & E1512: 201709 & -0.20 & Em & 45.4 \\
\hline & & & & & E1512: 201721 & -0.08 & Em & 21.2 \\
\hline \multirow[t]{2}{*}{ p09 } & 0.34 & 263 & 9.1 & int? & E1621: 102127 & 0.38 & no & $<5$ \\
\hline & & & & & E1621: 102139 & 0.35 & no & $<5$ \\
\hline \multirow[t]{2}{*}{$\mathrm{p} 10$} & 0.40 & 211 & 8.6 & int? & E1621: 301135 & 0.44 & no & $<5$ \\
\hline & & & & & E1621: 301126 & 0.50 & no & $<5$ \\
\hline \multirow[t]{2}{*}{$\mathrm{p} 11$} & 0.37 & 216 & 18.8 & no & E1621: 300268 & 0.36 & Em? & $<5$ \\
\hline & & & & & E1621: 300251 & 0.48 & no & $<5$ \\
\hline \multirow[t]{2}{*}{$\mathrm{p} 12$} & 0.27 & 212 & 9.5 & int? & E1621: 301023 & 0.53 & no & $\ldots$ \\
\hline & & & & & E1621: 301020 & 0.38 & no & $\ldots$ \\
\hline \multirow[t]{2}{*}{$\mathrm{p} 13$} & 0.53 & 78 & 14.6 & no & E1621: 300307 & 0.65 & no & $\ldots$ \\
\hline & & & & & E1621: 300306 & 0.54 & no & $<5$ \\
\hline
\end{tabular}

a Image classification. Pairs are classified as interacting (int), possibly interacting (int?), or not interacting (no).

${ }^{\mathrm{b}}$ Using $g-r k$-corrections from YEC (using spectra of CWW).

${ }^{c}$ CNOC1 spectral classification. Galaxy spectra are classified as having emission lines (Em), possibly having emission lines (Em?), or not having emission lines (no). 